Algebraic $\& \mathcal{G}$ Geometric Topology

Volume 1 (2001) 201-230

Published: 14 April 2001

ATG

\title{
Filtered Topological Cyclic Homology and relative $K$-theory of nilpotent ideals
}

\author{
Morten Brun
}

\begin{abstract}
In this paper certain filtrations of topological Hochschild homology and topological cyclic homology are examined. As an example we show how the filtration with respect to a nilpotent ideal gives rise to an ana$\log$ of a theorem of Goodwillie saying that rationally relative $K$-theory and relative cyclic homology agree. Our variation says that the $p$-torsion parts agree in a range of degrees. We use it to compute $K_{i}\left(\mathbb{Z} / p^{n}\right)$ for $i \leq p-3$.
\end{abstract}

AMS Classification 19D55; 19D50, 55P42

Keywords $K$-theory, topological Hochschild homology, cyclic homology, topological cyclic homology

\section{Introduction}

The aim of this paper is to examine a certain filtration of topological Hochschild homology of a functor with smash product equipped with a filtration. The former filtration preserves the cyclic structure and it induces a filtration of topological cyclic homology. By a theorem of McCarthy [19] topological cyclic homology is closely related to algebraic $K$-theory, and in some interesting cases topological cyclic homology determines the $K$-groups. The methods developed in this paper stem from a paper of Hesselholt and Madsen, where the $K$-groups for finite algebras over Witt vectors of perfect fields of positive characteristic are computed [13]. One difference is that here general filtrations are considered, while the filtrations considered in [13] are split. In the paper [7] the filtration of $\mathbb{Z} / p^{n}$ by the powers of the ideal $p \mathbb{Z} / p^{n}$ was used to compute topological Hochschild homology of the ring $\mathbb{Z} / p^{n}$. This is the example that motivated the generality of the present paper.

Given a ring $R$ with an ideal $I$, we shall let $K(R, I)$ denote the homotopy fibre of the map $K(R) \rightarrow K(R / I)$, and we shall let $H C(R, I)$ denote the homotopy fibre of the map $H C(R) \rightarrow H C(R / I)$. As an example of how the filtrations constructed can be useful, we prove the following analog of a theorem of Goodwillie [11]. 
Theorem 6.1 Let $R$ be a simplicial ring with an ideal $I$ satisfying $I^{m}=$ 0 . Suppose that $R$ and $R / I$ are flat as modules over $\mathbb{Z}$. Then there is an isomorphism of homotopy groups of $p$-adic completions

$$
\pi_{i} K(R, I)_{p}^{\wedge} \cong \pi_{i-1} H C(R, I)_{p}^{\wedge}
$$

when $0 \leq i<p /(m-1)-2$ and a surjection

$$
\pi_{i} K(R, I)_{p}^{\wedge} \rightarrow \pi_{i-1} H C(R, I)_{p}^{\wedge}
$$

when $i<p /(m-1)-1$.

In the case where $R$ and $R / I$ are not flat as modules over $\mathbb{Z}$, we can replace them with weakly equivalent simplicial rings that are degreewise free abelian groups. Since $K(R, I)$ is homotopy invariant we obtain that $K_{i}(R, I)_{p}^{\wedge}$ is isomorphic to the $p$-adic completion of derived relative cyclic homology in the same range of degrees. In section 7 we recall the definition of derived cyclic homology, and we compute enough derived cyclic homology groups for $\mathbb{Z} / p^{n}$ to deduce the following result.

Corollary 7.4 For $1 \leq i \leq p-3$, the $K$-groups of $\mathbb{Z} / p^{n}$ are:

$$
\pi_{i} K\left(\mathbb{Z} / p^{n}\right) \cong \begin{cases}0 & \text { if } i \text { is even } \\ \mathbb{Z} / p^{j(n-1)}\left(p^{j}-1\right) & \text { if } i=2 j-1\end{cases}
$$

The starting point of the above result is Quillen's computation of $\pi_{*} K(\mathbb{Z} / p)$ in [23]. The result agrees with the computation of $K_{i}\left(\mathbb{Z} / p^{n}\right)$ for $0 \leq i \leq 4$ of Aisbett, Puebla and Snaith [1] starting from Evens and Friedlander's computation of $K_{i}\left(\mathbb{Z} / p^{2}\right)$ for $0 \leq i \leq 4$ and for $p \geq 5$ [10]. It also shows that the homotopy groups of $B G L\left(\mathbb{Z} / p^{n}\right)^{+}$and of the homotopy fibre of $\psi^{p^{n}}-\psi^{p^{n-1}}: B U \rightarrow B U$ are different so these spaces can not be homotopy equivalent, as was also proven by Priddy in [21] in the case $n=2$.

In view of Quillen's computation of $\pi_{*} K(\mathbb{Z} / p)$ only the $p$-torsion part of corollary 7.4 is hard to prove. Let us show that if $l$ is relatively prime to $p$ then the natural map $K\left(\mathbb{Z} / p^{n}\right) \rightarrow K(\mathbb{Z} / p)$ induces an isomorphism on homotopy groups with coefficients in $\mathbb{Z} / l$. Since $B G L\left(\mathbb{Z} / p^{n}\right)^{+}$and $B G L(\mathbb{Z} / p)^{+}$are simple spaces it suffices by the Whitehead theorem to show that the map $B G L\left(\mathbb{Z} / p^{n}\right)^{+} \rightarrow B G L\left(\mathbb{Z} / p^{n-1}\right)^{+}$induces an isomorphism on homology with coefficients in $\mathbb{Z} / l$. For this we note that the kernel of the map $G L_{m}\left(\mathbb{Z} / p^{n}\right) \rightarrow$ $G L_{m}\left(\mathbb{Z} / p^{n-1}\right)$ consists of matrices of the form $I+p^{n-1} M$. Multiplication is given by $\left(I+p^{n-1} M\right)\left(I+p^{n-1} N\right)=I+p^{n-1}(M+N)$ so the kernel $J$ of the 
map $G L\left(\mathbb{Z} / p^{n}\right) \rightarrow G L\left(\mathbb{Z} / p^{n-1}\right)$ is a vectorspace over $\mathbb{Z} / p$. The Serre spectral sequence

$$
H_{*}\left(B G L\left(\mathbb{Z} / p^{n-1}\right), H_{*}(B J, \mathbb{Z} / l)\right) \Rightarrow H_{*}\left(B G L\left(\mathbb{Z} / p^{n}\right), \mathbb{Z} / l\right)
$$

associated to the fibration $B J \rightarrow B G L\left(\mathbb{Z} / p^{n}\right) \rightarrow B G L\left(\mathbb{Z} / p^{n-1}\right)$ collapses to an isomorphism $H_{*}\left(B G L\left(\mathbb{Z} / p^{n-1}, \mathbb{Z} / l\right) \cong H_{*}\left(B G L\left(\mathbb{Z} / p^{n}\right), \mathbb{Z} / l\right)\right.$.

Only elementary properties of the filtrations of topological Hochschild homology and topological cyclic homology are studied in this note. The focus is on a filtered version of the norm cofibration sequence for the fixed points of topological Hochschild homology. Traditionally, for example in [4] and in [13], the role of the norm cofibration sequence is that it allows one to determine the fixed point spectra inductively. Here we use it to keep track of the connectivity properties of our filtration of topological cyclic homology.

The paper is organized as follows: In section 2 generalities on filtrations of monoids in a symmetric monoidal category are given. It is noted that a filtered monoid is a monoid in the symmetric monoidal category of filtered objects, and therefore it fits into the Hochschild construction. In section 3 a filtered functor with smash product is defined to be a filtered monoid in the category of Gamma spaces, and fundamental properties of the topological Hochschild homology of a filtered functor with smash product are established. In section 4 we introduce the concept of a cyclotomic filtered Gamma space. This is a filtered Gamma space with an action of the circle group having enough extra properties to make it possible to construct a filtered version of topological cyclic homology out of it. It is shown that topological Hochschild homology of a filtered functor with smash product is such a a cyclotomic filtered Gamma space. In section 6 a proof of theorem 6.1 is given. In section 7 we compute enough derived cyclic homology of the ring $\mathbb{Z} / p^{n}$ to prove corollary 7.4.

It might be appropriate add a remark on terminology. Following Bousfield and Friedlander [5] we do not assume Gamma spaces to be special, and we do not assume spectra to to be omega-spectra.

Acknowledgments This work was funded by a Marie Curie Fellowship. The author wishes to thank F. Waldhausen and the University of Bielefeld for hospitality while this paper was written and he wants to thank M. Bökstedt for his constant interest in and collaboration on the computations motivating the present work. In particular Bökstedt proved proposition 6.4 and proposition 7.2 before the author. His proofs are different from the ones presented here. Finally the author wants to thank the referee for a lot of helpful comments. 


\section{The Filtered Hochschild Construction}

In this section we shall study filtered objects in a category $\mathcal{C}$.

\section{$2.1 \quad$ Filtered Objects}

A filtered object in a category $\mathcal{C}$ is a functor from the category $\mathbb{Z}$, with exactly one morphism $n \rightarrow m$ if $n \leq m$, to $\mathcal{C}$. That is, a filtered object is a sequence

$$
\cdots \rightarrow X(-1) \rightarrow X(0) \rightarrow X(1) \rightarrow \cdots \rightarrow X(n) \rightarrow X(n+1) \rightarrow \cdots
$$

of composable morphisms in $\mathcal{C}$. A morphism of filtered objects is simply a natural transformation. For some choices of $\mathcal{C}$ there is a functor $H$ from the category $\mathcal{C}^{\mathbb{Z}}$ of filtered objects in $\mathcal{C}$ to the category of exact couples of (graded) abelian groups in the sense of Massey [18].

Example 2.1 Functors from filtered objects to exact couples:

(1) The category of chain complexes and injective chain homomorphisms together with the functor $H$ given by homology.

(2) We can take $\mathcal{C}$ to be the category of topological spaces and cofibrations and let $H$ be given by (generalized) homology.

Given objects $X_{i}$ in $\mathcal{C}$ we shall denote their coproduct by $\bigvee_{i} X_{i}$, and given a diagram $Z \leftarrow X \rightarrow Y$ we shall denote the colimit, that is, the pushout by $Z \cup_{X} Y$.

Lemma 2.2 Given a functor $F: \mathbb{Z} \times \mathbb{Z} \rightarrow \mathcal{C}$ and $k \in \mathbb{Z}$ the following diagram is a pushout diagram:

$$
\begin{array}{ccc}
\bigvee_{i+j=k} F(i-1, j) \cup_{F(i-1, j-1)} F(i, j-1) & \rightarrow \underset{i+j \leq k-1}{\operatorname{colim}} F(i, j) \\
\downarrow & & \downarrow \\
\bigvee_{i+j=k} F(i, j) & \rightarrow & \operatorname{colim}_{i+j \leq k} F(i, j) .
\end{array}
$$

\subsection{Filtered objects in monoidal categories}

From now on $\mathcal{C}=(\mathcal{C}, \otimes, I)$ shall denote a cocomplete symmetric monoidal category. Given filtered objects $X$ and $Y$ in $\mathcal{C}$ we can define a filtered object $X \otimes Y$ in $\mathcal{C}$ by letting $(X \otimes Y)(k)=\operatorname{colim}_{i+j \leq k} X(i) \otimes Y(j)$. The pairing $\otimes:$ 
$\mathcal{C}^{\mathbb{Z}} \times \mathcal{C}^{\mathbb{Z}} \rightarrow \mathcal{C}^{\mathbb{Z}}$ defines a symmetrical monoidal structure on $\mathcal{C}^{\mathbb{Z}}$ with unit $I$ given by the filtered object with $I(k)$ equal to the initial object in $\mathcal{C}$ for $k<0$ and with $I(k)$ equal to the unit for the monoidal structure of $\mathcal{C}$ when $k \geq 0$. We have consciously chosen the same symbols for the pairing and unit in $\mathcal{C}^{\bar{Z}}$ as in $\mathcal{C}$ because we can consider $\mathcal{C}$ as a full symmetric monoidal subcategory of $\mathcal{C}^{\mathbb{Z}}$

A filtered monoid in $\mathcal{C}$ is a monoid in the category $\mathcal{C}^{\mathbb{Z}}$. Explicitly, a filtered monoid in $\mathcal{C}$ is a sequence

$$
\cdots \rightarrow M(-1) \rightarrow M(0) \rightarrow M(1) \rightarrow \cdots \rightarrow M(n) \rightarrow M(n+1) \rightarrow \cdots
$$

of composable morphisms in $\mathcal{C}$ together with morphisms

$$
\begin{gathered}
\mu_{i, j}: M(i) \otimes M(j) \rightarrow M(i+j) \\
\eta: I \rightarrow M(0),
\end{gathered}
$$

satisfying the following relations for associativity and unitality:

$$
\begin{gathered}
\mu_{i+j, k} \circ\left(\mu_{i, j} \otimes \operatorname{id}_{M(k)}\right)=\mu_{i, j+k} \circ\left(\operatorname{id}_{M(i)} \otimes \mu_{j, k}\right), \\
\mu_{0, i} \circ\left(\eta \otimes \operatorname{id}_{M(i)}\right)=\lambda_{M(i)}, \\
\mu_{i, 0} \circ\left(\operatorname{id}_{M(i)} \otimes \eta\right)=\rho_{M(i)} .
\end{gathered}
$$

Here $\lambda_{M(i)}: I \otimes M(i) \cong M(i)$ and $\rho_{M(i)}: M(i) \otimes I \cong M(i)$ are part of the symmetric monoidal structure of $\mathcal{C}$. We shall call a filtered monoid in the category of abelian groups a filtered ring.

If $*$ is a terminal object of $\mathcal{C}$ and $X \rightarrow Y$ is a map in $\mathcal{C}$, we shall denote any choice of pushout of the diagram $* \leftarrow X \rightarrow Y$ by $Y / X$. We shall say that the product $\otimes$ of $\mathcal{C}$ commutes with quotients if there is a natural isomorphism $\left(X_{1} / X_{2}\right) \otimes Y \cong\left(X_{1} \otimes Y\right) /\left(X_{2} \otimes Y\right)$.

Lemma 2.3 If $\mathcal{C}$ is a cocomplete symmetric monoidal category with a terminal object, then given filtered objects $X$ and $Y$ of $\mathcal{C}$ there is an isomorphism:

$$
\frac{(X \otimes Y)(k)}{(X \otimes Y)(k-1)} \cong \bigvee_{i+j=k} \frac{X(i) \otimes Y(j)}{X(i-1) \otimes Y(j) \cup_{X(i-1) \otimes Y(j-1)} X(i) \otimes Y(j-1)}
$$

If in addition $\otimes$ commutes with quotients, then there is an isomorphism:

$$
\frac{(X \otimes Y)(k)}{(X \otimes Y)(k-1)} \cong \bigvee_{i+j=k} \frac{X(i)}{X(i-1)} \otimes \frac{Y(j)}{Y(j-1)}
$$


Proof For the first part, it suffices to note that by lemma 2.2 the following diagram in $\mathcal{C}$ is a pushout:

$$
\begin{array}{ccc}
\bigvee_{i+j=k} X(i-1) \otimes Y(j) \cup_{X(i-1) \otimes Y(i-1)} X(i) \otimes Y(j-1) & \rightarrow & (X \otimes Y)(k-1) \\
\downarrow & & \downarrow \\
\bigvee_{i+j=k} X(i) \otimes Y(j) & & (X \otimes Y)(k) .
\end{array}
$$

For the second part, we note that:

$$
\left(X_{1} / X_{0}\right) \otimes\left(Y_{1} / Y_{0}\right) \cong \frac{\left(X_{1} / X_{0}\right) \otimes Y_{1}}{\left(X_{1} / X_{0}\right) \otimes Y_{0}} \cong \frac{\left(X_{1} \otimes Y_{1}\right) /\left(X_{0} \otimes Y_{1}\right)}{\left(X_{1} \otimes Y_{0}\right) /\left(X_{0} \otimes Y_{0}\right)}
$$

for $X_{0} \rightarrow X_{1}$ and $Y_{0} \rightarrow Y_{1}$ maps in $\mathcal{C}$, and that given a map $B \cup_{D} C \rightarrow A$ in $\mathcal{C}$ we have:

$$
\frac{A / B}{C / D} \cong \frac{A}{B \cup_{D} C}
$$

\subsection{The Hochschild construction}

Let $\mathcal{C}$ denote a symmetric monoidal category. The Hochschild construction is a functor $Z$ from the category of monoids in $\mathcal{C}$ to Connes' category of cyclic objects in $\mathcal{C}$. A good reference for the category of cyclic objects is the book of Loday [15, chapter 6]. Given a monoid $M$ in $\mathcal{C}, Z(M)$ is defined as follows: It has $n$-simplices

$$
Z_{n}(M)=M \otimes \cdots \otimes M \quad(n+1) \text { factors. }
$$

The cyclic operator is given by the automorphism $t_{n}$ of $M \otimes \cdots \otimes M$ cyclically shifting the $(n+1)$ factors to the right. The face maps are given by the formula:

$$
d_{i}=t_{n-1}^{i} \circ(\mu \otimes \mathrm{id}) \circ t_{n}^{-i}, \quad 0 \leq i \leq n,
$$

where $\mu: M \otimes M \rightarrow M$ is the multiplication in $M$. The degeneracies are given by the formula:

$$
s_{i}=t_{n+1}^{(i+1)} \circ(\eta \otimes \mathrm{id}) \circ t_{n}^{-(i+1)}, \quad 0 \leq i \leq n,
$$

where $\eta: I \rightarrow M$ is the unit in $M$.

Since $\mathcal{C}^{\mathbb{Z}}$ is equipped with a symmetric monoidal structure, we can also consider the Hochschild construction on monoids in $\mathcal{C}^{\mathbb{Z}}$, that is on filtered monoids in $\mathcal{C}$.

Proposition 2.4 Let $M$ be a filtered monoid in a cocomplete symmetric monoidal category $\mathcal{C}$, where $\otimes$ commutes with quotients. Then for each $n \geq 0$ there is an isomorphism of cyclic objects

$$
\frac{Z_{n}(M)(k)}{Z_{n}(M)(k-1)} \cong \bigvee_{i_{0}+\cdots+i_{n}=k} \frac{M\left(i_{0}\right)}{M\left(i_{0}-1\right)} \otimes \cdots \otimes \frac{M\left(i_{n}\right)}{M\left(i_{n}-1\right)}
$$


Proof This is a direct consequence of lemma 2.3.

The above proposition can be reformulated in terms of the associated graded monoid $\operatorname{gr} M$ for $M$. Here gr $M$ is the filtered monoid in $\mathcal{C}$ with

$$
\operatorname{gr}(M)(k)=\bigvee_{i \leq k} M(i) / M(i-1)
$$

and with multiplication induced by the maps

$$
\begin{aligned}
\frac{M(i)}{M(i-1)} \otimes \frac{M(j)}{M(j-1)} & \cong \frac{M(i) \otimes M(j)}{M(i) \otimes M(j-1) \cup M(i-1) \otimes M(j-1)} M(i-1) \otimes M(j) \\
& \rightarrow \frac{M(i+j)}{M(i+j-1)} .
\end{aligned}
$$

The proposition says that the filtration quotients for $Z(M)$ and $Z(\operatorname{gr} M)$ are isomorphic.

\section{$3 \quad$ Filtered Topological Hochschild Homology}

\subsection{Topological Hochschild homology}

We briefly recall the definition of topological Hochschild homology: Let $I$ denote the category with one object $n$ for each integer $n \geq 0$ and with $I(m, n)$ given by the set of injective maps from $\{1, \ldots m\}$ to $\{1, \ldots, n\}$. Let $L$ denote a functor with smash product in the sense of Bökstedt [2] or in the more restrictive sense described below. $\operatorname{THH}(L)$ is the cyclic pointed simplicial set with $k$-simplices equal to the homotopy colimit

$$
\underset{\left(i_{0}, \ldots, i_{k}\right) \in I^{k+1}}{\operatorname{hocolim}} F\left(S^{i_{0}} \wedge \cdots \wedge S^{i_{k}}, L\left(S^{i_{0}}\right) \wedge \cdots \wedge L\left(S^{i_{k}}\right)\right)
$$

and with structure maps of the same type as for the Hochschild construction. Details on this construction can be found in [3]. The symbol $F$ denotes derived function space, that is, if $X$ and $Y$ are pointed simplicial sets, then $F(X, Y)=$ $\mathcal{S}_{*}(X, \sin |Y|)$, where $\sin |Y|$ denotes the singular complex on the geometric realization of $Y$, and $\mathcal{S}_{*}$ denotes the internal function object in the category $\mathcal{S}_{*}$ of pointed simplicial sets. Occasionally we shall write $\Omega^{n} Y=F\left(S^{n}, Y\right)$ for the $n$ 'th loop space of $Y$.

For the purpose of this note, a functor with smash product is a monoid in the category $\Gamma \mathcal{S}_{*}$ of Gamma spaces considered for example by Bousfield and Friedlander [5, definition 3.1]. Let us recall that a Gamma space is a pointed functor 
from the category $\Gamma$ of pointed finite sets to the category $\mathcal{S}_{*}$ of pointed simplicial sets. To be precise $\Gamma$ is the category with one object $n^{+}=\{0,1, \ldots, n\}$ for each $n \geq 0$, and with $\Gamma\left(m^{+}, n^{+}\right)$the set functions from $m^{+}$to $n^{+}$fixing 0 . A pointed category is a category with an object which is both initial and terminal, and a functor between pointed categories is pointed if it takes an object which is both initial and final to an object of the same kind. Let us stress that our notion of a Gamma space is different from the notion in the paper by Segal [24]. Given two Gamma spaces $X$ and $Y$, their smash product is the Gamma space $X \wedge Y$ with

$$
(X \wedge Y)\left(n^{+}\right)=\operatorname{colim}_{n_{1}^{+} \wedge n_{2}^{+} \rightarrow n^{+}} X\left(n_{1}^{+}\right) \wedge Y\left(n_{2}^{+}\right) .
$$

The unit for the operation $\wedge$ is the functor $\mathbb{S}: \Gamma \rightarrow \mathcal{S}_{*}$ with $\mathbb{S}\left(n^{+}\right)=n^{+}$. Lydakis noted in [16, Theorem 2.18] that the category of Gamma spaces is a symmetric monoidal category with respect to the smash product pairing and unit. By definition a functor with smash product $L$ is a monoid in the category $\Gamma \mathcal{S}_{*}$. Explicitly this means that $L$ is a pointed functor $L: \Gamma \rightarrow \mathcal{S}_{*}$ together with natural transformations

$$
\begin{aligned}
\mu: L\left(m^{+}\right) & \wedge L\left(n^{+}\right) \rightarrow L\left(m^{+} \wedge n^{+}\right), \\
\eta & : n^{+} \rightarrow L\left(n^{+}\right),
\end{aligned}
$$

satisfying the following relations for associativity and unitality:

$$
\mu \circ(\mu \wedge \mathrm{id})=\mu \circ(\mathrm{id} \wedge \mu), \quad \mu \circ(\eta \wedge \mathrm{id})=\lambda, \quad \mu \circ(\mathrm{id} \wedge \eta)=\rho,
$$

where $\lambda: m^{+} \wedge L\left(n^{+}\right) \rightarrow L\left(m^{+} \wedge n^{+}\right)$is adjoint to the map

$$
m^{+} \rightarrow \Gamma\left(n^{+}, m^{+} \wedge n^{+}\right) \stackrel{L}{\rightarrow} \mathcal{S}_{*}\left(L\left(n^{+}\right), L\left(m^{+} \wedge n^{+}\right)\right)
$$

and $\rho: L\left(m^{+}\right) \wedge n^{+} \rightarrow L\left(m^{+} \wedge n^{+}\right)$is adjoint to the map

$$
n^{+} \rightarrow \Gamma\left(m^{+}, m^{+} \wedge n^{+}\right) \stackrel{L}{\rightarrow} \mathcal{S}_{*}\left(L\left(m^{+}\right), L\left(m^{+} \wedge n^{+}\right)\right) .
$$

Example 3.1 For this note the most relevant example of an FSP is the functor $\widetilde{\mathbb{Z}}: \Gamma \rightarrow \mathcal{S}_{*}$ with $\widetilde{\mathbb{Z}}\left(n^{+}\right)=\mathbb{Z}\left\{n^{+}\right\} / \mathbb{Z}\{0\}$ the reduced free abelian group on the pointed set $n^{+}=\{0,1, \ldots, n\}$. The multiplication $\mu$ is given by the composition

$$
\mu: \widetilde{\mathbb{Z}}\left(m^{+}\right) \wedge \widetilde{\mathbb{Z}}\left(n^{+}\right) \rightarrow \widetilde{\mathbb{Z}}\left(m^{+}\right) \otimes_{\mathbb{Z}} \widetilde{\mathbb{Z}}\left(n^{+}\right) \cong \widetilde{\mathbb{Z}}\left(m^{+} \wedge n^{+}\right),
$$

and the unit is given by the inclusion of the basis $n^{+}$in the free abelian group $\mathbb{Z}\left\{n^{+}\right\}$composed with the quotient map. Given any ring $R$ we obtain an FSP $\widetilde{R}$ with $\widetilde{R}\left(n^{+}\right)=R \otimes \widetilde{\mathbb{Z}}\left(n^{+}\right)$. The multiplication and the unit in $\widetilde{R}$ are explained in example 3.6 below. 
Given a Gamma space $X$, we can extend it to a functor $X_{1}$ defined on the category of pointed sets by letting

$$
X_{1}(K)=\operatorname{colim}_{n^{+} \rightarrow K} X\left(n^{+}\right)
$$

for $K$ a pointed set, and we can define an endofunctor $X_{2}$ on $\mathcal{S}_{*}$ by letting $\left(X_{2}(U)\right)_{k}=\left(X_{1}\left(U_{k}\right)\right)_{k}$ for $U$ a pointed simplicial set. From now on we shall not distinguish notationally between a Gamma space and the induced endofunctor on $\mathcal{S}_{*}$.

Given a Gamma space $X$ and pointed simplicial sets $U$ and $V$, there is a map $X(U) \wedge V \rightarrow X(U \wedge V)$ obtained by applying the above map $\rho$ degreewise. The following lemma is given in [16, prop. 5.21]:

Lemma 3.2 If $U$ is $m$-connected and $V$ is $n$-connected, then the map $X(U) \wedge$ $V \rightarrow X(U \wedge V)$ is $2 m+n+3$-connected.

Together with the approximation lemma of Bökstedt (see either [2] or [7, lemma 2.5.1]), it can be used to prove the following.

Lemma 3.3 Given a Gamma space $X$ and $\left(j_{0}, \ldots, j_{k}\right) \in I^{k+1}$, then the map

$$
\begin{aligned}
F\left(S^{j_{0}} \wedge \cdots \wedge S^{j_{k}}, X\left(S^{j_{0}}\right) \wedge \cdots \wedge X\left(S^{j_{k}}\right)\right) & \rightarrow \\
& \\
\left(\begin{array}{l}
\text { hocolime } \\
\left(i_{0}, \ldots, i_{k}\right) \in I^{k+1}
\end{array}\right. & F\left(S^{i_{0}} \wedge \cdots \wedge S^{i_{k}}, X\left(S^{i_{0}}\right) \wedge \cdots \wedge X\left(S^{i_{k}}\right)\right)
\end{aligned}
$$

is $j-1$-connected. Here $j$ denotes the minimum of the cardinalities of $j_{0}, \ldots, j_{k}$.

Given an FSP $L$ and a finite pointed set $n^{+}$, we shall let $\operatorname{THH}\left(L ; n^{+}\right)$denote the cyclic pointed simplicial set with $k$-simplices equal to the homotopy colimit

$$
\underset{\left(i_{0}, \ldots, i_{k}\right) \in I^{k+1}}{\operatorname{hocolim}} F\left(S^{i_{0}} \wedge \cdots \wedge S^{i_{k}}, L\left(S^{i_{0}}\right) \wedge \cdots \wedge L\left(S^{i_{k}}\right) \wedge n^{+}\right),
$$

where $n^{+}$acts as a dummy variable for the cyclic structure. There is an endofunctor $\operatorname{THH}(L,-)$ on $\mathcal{S}_{*}$ associated to the Gamma space $n^{+} \mapsto \operatorname{THH}\left(L ; n^{+}\right)$. We shall freely use the identification $\operatorname{THH}(L) \cong \operatorname{THH}\left(L ; 1^{+}\right) \cong \operatorname{THH}\left(L ; S^{0}\right)$.

Lemma 3.4 The map $\operatorname{THH}(L) \rightarrow \Omega^{n} \operatorname{THH}\left(L ; S^{n}\right)$, adjoint to $\operatorname{THH}\left(S ; S^{0}\right) \wedge$ $S^{n} \rightarrow \operatorname{THH}\left(L ; S^{n}\right)$, is a weak equivalence.

Proof By the work of Segal [24, prop. 1.4] it suffices to show that the Gamma space $n^{+} \mapsto \operatorname{THH}\left(L ; n^{+}\right)$is very special, that is, the map

$$
\left(\operatorname{pr}_{1 *}, \operatorname{pr}_{2 *}\right): \operatorname{THH}\left(L ; m^{+} \vee n^{+}\right) \rightarrow \operatorname{THH}\left(L ; m^{+}\right) \times \operatorname{THH}\left(L ; n^{+}\right)
$$


induced by the projections $\operatorname{pr}_{1}: m^{+} \vee n^{+} \rightarrow m^{+} \vee 0^{+}=m^{+}$and $\operatorname{pr}_{2}: m^{+} \vee n^{+} \rightarrow$ $0^{+} \vee n^{+}=n^{+}$, is a weak equivalence, and that the monoid $\pi_{0} \operatorname{THH}\left(L ; 1^{+}\right)$ is a group. By lemma 3.5 below it suffices to show that the Gamma spaces $n^{+} \mapsto \mathrm{THH}_{k}\left(L ; n^{+}\right)$are very special. To see that the map

$$
\mathrm{THH}_{k}\left(L ; m^{+} \vee n^{+}\right) \rightarrow \mathrm{THH}_{k}\left(L ; m^{+}\right) \times \mathrm{THH}_{k}\left(L ; n^{+}\right)
$$

is a weak equivalence, it suffices by the approximation lemma 3.3 to note that by the Whitehead theorem the map

$$
\begin{aligned}
& L\left(S^{i_{0}}\right) \wedge \cdots \wedge L\left(S^{i_{k}}\right) \wedge\left(m^{+} \vee n^{+}\right) \\
& \cong\left(L\left(S^{i_{0}}\right) \wedge \cdots \wedge L\left(S^{i_{k}}\right) \wedge m^{+}\right) \vee\left(L\left(S^{i_{0}}\right) \wedge \cdots \wedge L\left(S^{i_{k}}\right) \wedge n^{+}\right) \\
& \rightarrow\left(L\left(S^{i_{0}}\right) \wedge \cdots \wedge L\left(S^{i_{k}}\right) \wedge m^{+}\right) \times\left(L\left(S^{i_{0}}\right) \wedge \cdots \wedge L\left(S^{i_{k}}\right) \wedge n^{+}\right)
\end{aligned}
$$

is $2\left(i_{0}+\cdots+i_{k}\right)-1$-connected.

To see that $\pi_{0} \mathrm{THH}_{k}\left(L ; 1^{+}\right)$is a group, it suffices to note that $\pi_{0} F\left(S^{i_{0}} \wedge \cdots \wedge\right.$ $\left.S^{i_{k}}, L\left(S^{i_{0}}\right) \wedge \cdots \wedge L\left(S^{i_{k}}\right)\right)$ is a group.

We owe the following lemma to S. Schwede.

Lemma 3.5 Let $X$ be a simplicial Gamma space, and assume that for each $k, X_{k}$ is a very special Gamma space. Then the Gamma topological space $|X|$ sending $n^{+}$to the realization of $[k] \mapsto X_{k}\left(n^{+}\right)$is very special.

Proof It follows from the realization lemma and the fact that realization commutes with products that the resulting Gamma space is special, that is, the map $\left|X\left(m^{+} \vee n^{+}\right)\right| \rightarrow\left|X\left(m^{+}\right)\right| \times\left|X\left(n^{+}\right)\right|$induced by the projections $\mathrm{pr}_{1}$ and $\mathrm{pr}_{2}$ is a homotopy equivalence. A special Gamma space $Y$ is very special when the monoid $\pi_{0}\left|Y\left(1^{+}\right)\right|$with multiplication induced by the composite

$$
\left|Y\left(1^{+}\right)\right| \times\left|Y\left(1^{+}\right)\right| \stackrel{f}{\rightarrow}\left|Y\left(2^{+}\right)\right| \stackrel{Y(\mu)}{\rightarrow}\left|Y\left(1^{+}\right)\right|
$$

is a group. Here $\mu: 2^{+} \rightarrow 1^{+}$is the fold map with $\mu(i)=1$ for $i=1,2$ and $f$ is a homotopy inverse to the homotopy equivalence $\left|Y\left(2^{+}\right)\right| \rightarrow\left|Y\left(1^{+}\right)\right| \times\left|Y\left(1^{+}\right)\right|$. This is equivalent to the map $\left(Y(\mu), Y\left(\mathrm{pr}_{2}\right)\right):\left|Y\left(2^{+}\right)\right| \rightarrow\left|Y\left(1^{+}\right)\right| \times\left|Y\left(1^{+}\right)\right|$ being a homotopy equivalence. (Clearly if this map is a homotopy equivalence, then $\pi_{0} Y\left(1^{+}\right)$is a group. Conversely, if $\pi_{0} Y\left(1^{+}\right)$is a group, then this map induces an isomorphism on $\pi_{n}$ for all $n \geq 0$, and by the Whitehead theorem we can conclude that it is a homotopy equivalence.) It follows from the realization lemma that $|X|$ is very special. 


\subsection{Filtered Topological Hochschild Homology}

To make a filtered version of topological Hochschild homology we replace the category $\mathcal{S}_{*}$ of pointed simplicial sets by the category $\mathcal{S}_{*}{ }^{\mathbb{Z}}$ of filtered pointed simplicial sets. By a Gamma filtered space we shall mean a pointed functor from $\Gamma$ to $\mathcal{S}_{*}{ }^{\mathbb{Z}}$. The smash product of two Gamma filtered spaces $X$ and $Y$, given by the formula

$$
(X \wedge Y)\left(n^{+}\right)=\operatorname{colim}_{\substack{n_{1}^{+} \wedge n_{2}^{+} \rightarrow n^{+}}} X\left(n_{1}^{+}\right) \wedge Y\left(n_{2}^{+}\right)
$$

makes the category $\Gamma \mathcal{S}_{*}^{\mathbb{Z}}$ of Gamma filtered spaces into a symmetric monoidal category. A filtered $F S P$ is a monoid in the category $\Gamma \mathcal{S}_{*}{ }^{\mathbb{Z}}$. Explicitly a filtered FSP can be described as a functor $L: \Gamma \times \mathbb{Z} \rightarrow \mathcal{S}_{*}$ together with natural transformations

$$
\begin{gathered}
\mu: L\left(m^{+}, s\right) \wedge L\left(n^{+}, t\right) \rightarrow L\left(m^{+} \wedge n^{+}, s+t\right) \\
\eta: n^{+} \rightarrow L\left(n^{+}, 0\right)
\end{gathered}
$$

satisfying the following relations:

$$
\mu \circ(\mu \wedge \mathrm{id})=\mu \circ(\mathrm{id} \wedge \mu), \quad \mu \circ(\eta \wedge \mathrm{id})=\lambda, \quad \mu \circ(\mathrm{id} \wedge \eta)=\rho,
$$

where $\lambda: m^{+} \wedge L\left(n^{+}, s\right) \rightarrow L\left(m^{+} \wedge n^{+}, s\right)$ is adjoint to the map

$$
m^{+} \rightarrow \Gamma\left(n^{+}, m^{+} \wedge n^{+}\right) \stackrel{L(-, s)}{\rightarrow} \mathcal{S}_{*}\left(L\left(n^{+}, s\right), L\left(m^{+} \wedge n^{+}, s\right)\right)
$$

and $\rho: L\left(m^{+}, s\right) \wedge n^{+} \rightarrow L\left(m^{+} \wedge n^{+}, s\right)$ is adjoint to the map

$$
n^{+} \rightarrow \Gamma\left(m^{+}, m^{+} \wedge n^{+}\right) \stackrel{L(-, s)}{\rightarrow} \mathcal{S}_{*}\left(L\left(m^{+}, s\right), L\left(m^{+} \wedge n^{+}, s\right)\right) .
$$

Note that the category of Gamma filtered spaces is isomorphic to the category of filtered Gamma spaces, and hence a filtered FSP also can be described as being a filtered monoid in the category of Gamma spaces.

Example 3.6 Given a filtered ring $R$ (that is, a filtered monoid in the category of abelian groups) there is a filtered FSP $\widetilde{R}$ with $\widetilde{R}\left(n^{+}, s\right)=\widetilde{\mathbb{Z}}\left(n^{+}\right) \otimes_{\mathbb{Z}} R(s)$. The multiplication is given by the composition

$$
\begin{aligned}
\mu: \widetilde{R}\left(m^{+}, s\right) \wedge \widetilde{R}\left(n^{+}, t\right) & \rightarrow \widetilde{R}\left(m^{+}, s\right) \otimes_{\mathbb{Z}} \widetilde{R}\left(n^{+}, t\right) \\
& \cong \widetilde{\mathbb{Z}}\left(m^{+} \wedge n^{+}\right) \otimes_{\mathbb{Z}} R(s) \otimes_{\mathbb{Z}} R(t) \\
& \rightarrow \widetilde{\mathbb{Z}}\left(m^{+} \wedge n^{+}\right) \otimes_{\mathbb{Z}} R(s+t) \\
& =\widetilde{R}\left(m^{+} \wedge n^{+}, s+t\right),
\end{aligned}
$$


induced by the multiplication in $R$ and the unit is given by the composition

$$
\eta: n^{+} \rightarrow \widetilde{\mathbb{Z}}\left(n^{+}\right) \rightarrow \widetilde{\mathbb{Z}}\left(n^{+}\right) \otimes_{\mathbb{Z}} R(0)=\widetilde{R}\left(n^{+}, 0\right)
$$

where the last map is induced from the unit of $R$.

The topological Hochschild homology of a filtered FSP $L$ is the filtered pointed simplicial set $\operatorname{THH}(L)$ with $k$-simplices of $\operatorname{THH}(L)(s)$ given by the homotopy colimit

$$
\underset{\left(i_{0}, \ldots, i_{k}\right) \in I^{k+1}}{\operatorname{hoc} \lim } F\left(S^{i_{0}} \wedge \cdots \wedge S^{i_{k}},\left(L\left(S^{i_{0}}\right) \wedge \cdots \wedge L\left(S^{i_{k}}\right)\right)(s)\right),
$$

where the smash product of the $L\left(S^{i_{\alpha}}\right)$ 's is a smash product of filtered pointed simplicial sets, and with cyclic structure of Hochschild type. We define cyclic spaces $\overline{\mathrm{THH}}(L, s)$ for $s \in \mathbb{Z}$ with $k$-simplices given by the homotopy colimit

$$
\underset{\left(i_{0}, \ldots, i_{k}\right) \in I^{k+1}}{\operatorname{hocolim}} F\left(S^{i_{0}} \wedge \cdots \wedge S^{i_{k}}, \frac{\left(L\left(S^{i_{0}}\right) \wedge \cdots \wedge L\left(S^{i_{k}}\right)\right)(s)}{\left(L\left(S^{i_{0}}\right) \wedge \cdots \wedge L\left(S^{i_{k}}\right)\right)(s-1)}\right),
$$

and with cyclic structure as for the Hochschild construction.

Of course there is also a filtered version of the Gamma space $n^{+} \mapsto \operatorname{THH}\left(L ; n^{+}\right)$ with $k$-simplices of $\operatorname{THH}\left(L ; n^{+}\right)(s)$ given by the homotopy colimit:

$$
\underset{\left(i_{0}, \ldots, i_{k}\right) \in I^{k+1}}{\operatorname{hocolim}} F\left(S^{i_{0}} \wedge \cdots \wedge S^{i_{k}},\left(L\left(S^{i_{0}}\right) \wedge \cdots \wedge L\left(S^{i_{k}}\right)\right)(s) \wedge n^{+}\right),
$$

and there is a Gamma space $n^{+} \mapsto \overline{\mathrm{THH}}\left(L, s ; n^{+}\right)$where the $k$-simplices of $\overline{\mathrm{THH}}\left(L, s ; n^{+}\right)$are given by the homotopy colimit

$$
\underset{\left(i_{0}, \ldots, i_{k}\right) \in I^{k+1}}{\operatorname{hocolim}} F\left(S^{i_{0}} \wedge \cdots \wedge S^{i_{k}}, \frac{\left(L\left(S^{i_{0}}\right) \wedge \cdots \wedge L\left(S^{i_{k}}\right)\right)(s)}{\left(L\left(S^{i_{0}}\right) \wedge \cdots \wedge L\left(S^{i_{k}}\right)\right)(s-1)} \wedge n^{+}\right) .
$$

Lemma 3.7 Let $X$ be a Gamma space satisfying that $X\left(n^{+}\right)$is l-connected for every $n \geq 0$. If $U$ is $m$-connected and $V$ is $n$-connected, then the map $X(U) \wedge V \rightarrow X(U \wedge V)$ is $2 m+n+l+3$-connected provided that $l, m$ and $n$ are $>1$.

Proof We consider the cofibre $X(U \wedge V) / X(U) \wedge V$ as a bisimplicial set $i, j \mapsto$ $Z_{i j}=X\left(U_{i} \wedge V_{i}\right)_{j} / X\left(U_{i}\right)_{j} \wedge V_{i}$. Since the cofibre of a cofibration of $l$-connected spaces is $l$-connected $Z_{i}$. is $l$-connected for every $i$, and by lemma $3.3 Z_{\cdot j}$ is $2 m+n+3$-connected for every $j$. Using the spectral sequence of BousfieldFriedlander [5, thm. B.5] we obtain the assertion of the lemma.

Together with the approximation lemma of Bökstedt ([2] or [7, lemma 2.5.1]) the above lemma proves the following. 
Lemma 3.8 Given a pointed functor $X: \Gamma^{k+1} \rightarrow \mathcal{S}_{*}$ and $\left(j_{0}, \ldots, j_{k}\right) \in I^{k+1}$ the map

$$
\begin{aligned}
& F\left(S^{j_{0}} \wedge \cdots \wedge S^{j_{k}}, X\left(S^{j_{0}}, \ldots, S^{j_{k}}\right)\right) \rightarrow \\
& \qquad \begin{array}{c}
\text { hocolimi } \\
\left(i_{0}, \ldots, i_{k}\right) \in I^{k+1}
\end{array} \\
&
\end{aligned}
$$

is $j-1$-connected, where $j$ denotes the minimum of $j_{0}, \ldots, j_{k}$.

Lemma 3.9 The spectra $n \mapsto \operatorname{THH}\left(L ; S^{n}\right)(s)$ and $n \mapsto \overline{\operatorname{THH}}\left(L, s ; S^{n}\right)$ are $\Omega$-spectra.

Proof Let us note that lemma 3.7 gives that $\left(L\left(S^{i_{0}}\right) \wedge \cdots \wedge L\left(S^{i_{k}}\right)\right)(s)$ is $i_{0}+$ $\cdots+i_{k}-1$-connected. Replacing lemma 3.3 by lemma 3.8 the proof of lemma 3.4 also proves this lemma.

We shall say that a filtered FSP $L$ is filtered by cofibrations if for every $X$ and $s$ the map $L(X)(s-1) \rightarrow L(X)(s)$ is a cofibration.

Lemma 3.10 Let $L$ be a filtered FSP, filtered by cofibrations. Then the map from $\operatorname{THH}(L)(s-1)$ to the homotopy fibre of the map $q: \operatorname{THH}(L)(s) \rightarrow$ $\overline{\mathrm{THH}}(L, s)$ is a weak equivalence.

Proof Let us start by showing that the map from the mapping cone of the $\operatorname{map} \mathrm{THH}_{k}\left(L ; S^{n}\right)(s-1) \rightarrow \mathrm{THH}_{k}\left(L ; S^{n}\right)(s)$ to $\overline{\mathrm{THH}}_{k}\left(L ; S^{n}, s\right)$ is $2(n-1)$ connected. If $X \rightarrow Y$ is a cofibration of $i+n$-connected pointed simplicial sets then by applying the Blakers-Massey theorem [25, p. 366] several times we see that the map from the mapping cone of the map $F\left(S^{i}, X\right) \rightarrow F\left(S^{i}, Y\right)$ to $F\left(S^{i}, Y / X\right)$ is $2(n-1)$-connected. Since the mapping cone construction commutes with geometric realization it follows that the map from the mapping cone of the map $\mathrm{THH}_{k}\left(L ; S^{n}\right)(s-1) \rightarrow \mathrm{THH}_{k}\left(L ; S^{n}\right)(s)$ to $\overline{\mathrm{THH}}_{k}\left(L ; S^{n}, s\right)$ is $2(n-1)$-connected.

Now let $q\left(S^{n}\right)$ denote the map $\operatorname{THH}\left(L ; S^{n}\right)(s) \rightarrow \overline{\operatorname{THH}}\left(L, s ; S^{n}\right)$, and let $h F q\left(S^{n}\right)$ denote its homotopy fibre. It then follows from the Blakers-Massey theorem that the map $\operatorname{THH}\left(L ; S^{n}\right)(s-1) \rightarrow h F q\left(S^{n}\right)$ is $2 n-4$-connected. From the weak equivalence $F\left(S^{n}, h F q\left(S^{n}\right)\right) \simeq h F q$ it follows that the map $\operatorname{THH}(L)(s-1) \rightarrow h F q$ is $n-4$-connected. Since $n$ is arbitrary, it follows that this map is a weak equivalence. 
Remark 3.11 Given a filtered FSP $L$ there is an FSP $L(0) / L(-1)$ taking $X$ to $L(X)(0) / L(X)(-1)$. If $L(s)=L(0)$ when $s \geq 0$ then by proposition 2.3 $\overline{\mathrm{THH}}(L, 0) \cong \mathrm{THH}(L(0) / L(-1))$ and $\mathrm{THH}(L)(0)=\mathrm{THH}(L(0))$. In this case the above lemma says that $\mathrm{THH}(L)(-1)$ is weakly equivalent to the homotopy fibre of the map $\operatorname{THH}(L(0)) \rightarrow \operatorname{THH}(L(0) / L(-1))$.

\section{Cyclotomic structure}

In this section we shall describe how the filtration on topological Hochschild homology of an FSP filtered by cofibrations is compatible with topological cyclic homology. We have based our presentation on the elementary version of topological cyclic homology given in [3]. Alternatively we could use the cyclotomic spectra in the sense of Madsen [4]. Since we do not need them for the main result of this paper we have chosen the technically simpler version of $T C$.

\subsection{Gamma epicyclic spaces}

Let us recall Goodwillie's notion of an epicyclic space from [12].

Definition 4.1 An epicyclic space is a cyclic space $Y$ equipped with maps $r_{q}: Y_{q j-1}^{C_{q}} \rightarrow Y_{j-1}$ for all $q \geq 1$ and $j \geq 1$, satisfying:

(1) $r_{q}:\left(\operatorname{sd}_{q} Y\right)^{C_{q}} \rightarrow Y$ is cyclic.

(2) $r_{a} \circ r_{q}=r_{a q}:\left(\operatorname{sd}_{a q} Y\right)^{C_{a q}} \rightarrow Y$.

(3) $r_{1}$ is the identity.

Here $\operatorname{sd}_{q} Y$ denotes the $q$-fold edgewise subdivision of $Y$ with $j$-simplices $\left(\operatorname{sd}_{q} Y\right)_{j}=Y_{q j-1}$. For a treatment of edgewise subdivision we refer to [3]. The most important properties of edgewise subdivision are that there is a simplicial action of $C_{q}$ on $\operatorname{sd}_{q} Y$, that there is an action of $S^{1}$ on $\left|\operatorname{sd}_{q} Y\right|$ extending the simplicial action of $C_{q}$, that there is an $S^{1}$-isomorphism $\left|\operatorname{sd}_{q} Y\right| \cong|Y|$, and that $\operatorname{sd}_{a q}=\operatorname{sd}_{a} \operatorname{sd}_{q}$. Note that $r_{q}$ induces a $C_{a}$-equivariant map $\left(\operatorname{sd}_{a q} Y\right)^{C_{q}}=$ $\operatorname{sd}_{a}\left(\operatorname{sd}_{q} Y\right)^{C_{q}} \rightarrow \operatorname{sd}_{a} Y$ for any $a$.

Write $Y^{C_{a}}$ for the topological space $\left|\left(\operatorname{sd}_{a} Y\right)^{C_{a}}\right| \cong|Y|^{C_{a}}$. There is a map $f_{q}$ : $Y^{C_{a q}} \cong|Y|^{C_{a q}} \rightarrow|Y|^{C_{a}} \cong Y^{C_{a}}$ induced by inclusion of fixed points. We shall call this map the Frobenius map. The map $\left(\operatorname{sd}_{a q} Y\right)^{C_{q}}=\operatorname{sd}_{a}\left(\operatorname{sd}_{q} Y\right)^{C_{q}} \rightarrow \operatorname{sd}_{a} Y$ induces a map $r_{q}: Y^{C_{a q}}=\left|\left(\left(\operatorname{sd}_{a q} Y\right)^{C_{q}}\right)^{C_{a}}\right| \rightarrow\left|\left(\operatorname{sd}_{a} Y\right)^{C_{a}}\right|=Y^{C_{a}}$, and we will 
call $r_{q}$ the $q$ 'th restriction map. (Following Hesselholt and Madsen [13], in conflict with Goodwillie's terminology) The maps $r_{q^{\prime}}$ and $f_{q}$ commute, that is $f_{q} r_{q^{\prime}}=r_{q^{\prime}} f_{q}$.

Let us fix a prime $p$. The restriction and Frobenius maps induce maps $r, f$ : $\prod_{n \geq 0} Y^{C_{p^{n}}} \rightarrow \prod_{n \geq 0} Y^{C_{p^{n}}}$. We let $\operatorname{tr}(Y, p)$ denote the homotopy equalizer of $r$ and the identity. Since $r f=f r$, the map $f$ induces an endomorphism on $\operatorname{tr}(Y, p)$. We define $t c(Y, p)$ to be the homotopy equalizer of $f$ and the identity on $\operatorname{tr}(Y, p)$. Note that since homotopy limits commute we could equally well have interchanged the roles of $r$ and $f$ in the definition of $t c(Y, p)$.

Definition 4.2 A Gamma epicyclic space is a Gamma object in the category of epicyclic spaces.

The main example of a Gamma epicyclic space is topological Hochschild homology. The restriction map $r_{q}: \operatorname{sd}_{q} \operatorname{THH}\left(L, n^{+}\right)^{C_{q}} \rightarrow \operatorname{THH}\left(L, n^{+}\right)$is defined degreewise by the following chain of maps:

$$
\begin{aligned}
& \left(\operatorname{sd}_{q} \operatorname{THH}\left(L, n^{+}\right)\right)_{k}^{C_{q}} \cong \\
& \underset{\left(n_{0}, \ldots, n_{k}\right) \in I^{k+1}}{\operatorname{hocolim}} F\left(\left(S^{n_{0}} \wedge \cdots \wedge S^{n_{k}}\right)^{\wedge q},\left(L\left(S^{n_{0}}\right) \wedge \cdots \wedge L\left(S^{n_{k}}\right)\right)^{\wedge q} \wedge n^{+}\right)^{C_{q}} \rightarrow \\
& \left.\underset{\left(n_{0}, \ldots, n_{k}\right) \in I^{k+1}}{\operatorname{hocolim}} F\left(\left(\left(S^{n_{0}} \wedge \cdots \wedge S^{n_{k}}\right)^{\wedge q}\right)^{C_{q}},\left(L\left(S^{n_{0}}\right) \wedge \cdots \wedge L\left(S^{n_{k}}\right)\right)^{\wedge q} \wedge n^{+}\right)^{C_{q}}\right) \cong \\
& \underset{\left(n_{0}, \ldots, n_{k}\right) \in I^{k+1}}{\operatorname{hoc}} F\left(S^{n_{0}} \wedge \cdots \wedge S^{n_{k}}, L\left(S^{n_{0}}\right) \wedge \cdots \wedge L\left(S^{n_{k}}\right) \wedge n^{+}\right)= \\
& \operatorname{THH}\left(L, n^{+}\right)_{k} \text {. }
\end{aligned}
$$

The first isomorphism is due to the isomorphism $\left.\underset{I^{q(k+1)}}{\operatorname{hocolim}} Z\right)^{C_{q}} \cong \underset{I^{k+1}}{\operatorname{hocolim}} Z^{C_{q}}$. The second map is given by restriction to fixed points and the last isomorphism is induced by the point set isomorphism $\left(X^{\wedge q}\right)^{C_{q}} \cong X$.

Given a Gamma epicyclic space $X$, we obtain simplicial epicyclic spaces $X\left(S^{n}\right)$. We can view these as epicyclic spaces and consider the spaces $t c\left(X\left(S^{n}\right), p\right)$. In order to see that these spaces assemble to a spectrum, let us first note that $n \mapsto\left(X\left(S^{n}\right)\right)^{C_{a}} \cong\left|X\left(S^{n}\right)\right|^{C_{a}}$ is a spectrum because the category of spectra is closed under limits, and limits are constructed degreewise. Since the same remark applies to homotopy limits we have a spectrum $n \mapsto t c\left(X\left(S^{n}\right), p\right)$.

Definition 4.3 Let $X$ be a Gamma epicyclic space. Topological cyclic homology at the prime $p$ of $X$ is the spectrum $T C(X, p)$ with $n$ 'th space $T C(X, p)_{n}=$ $\operatorname{tc}\left(X\left(S^{n}\right), p\right)$. 
We shall write $T C(L, p)$ instead of $T C(\operatorname{THH}(L), p)$. Our definition of topological cyclic homology at the prime $p$ agrees with the definition of Bökstedt, Hsiang and Madsen [3, def. 5.12.] At this point it is clear that our version of $T C$ has the same underlying space as the one in [3]. To see that the deloopings agree we first note that our spectrum $T C(L, p)$ is stably equivalent to the ones in Goodwillies note [11] and in [13, definiton 4.1]. Next we appeal to [13, prop. 2.6.2.].

By a Gamma cyclic space we shall mean a Gamma object in the category of cyclic pointed spaces. Given a Gamma cyclic space $X$ and a closed subgroup $H$ of $S^{1}$ we shall let $X^{H}$ denote the spectrum $n \mapsto\left|X\left(S^{n}\right)\right|^{H}$. If $X$ is a Gamma epicyclic space the restriction and Frobenius maps $r_{r}, f_{q}: X\left(S^{n}\right)^{C_{a q}} \rightarrow$ $X\left(S^{n}\right)^{C_{a}}$ induce maps $R_{q}, F_{q}: X^{C_{a q}} \rightarrow X^{C_{a}}$ of spectra. Given a cyclic pointed space $Z$ we shall define a spectrum $Z \wedge{ }^{L} X$ by the formula

$$
\left(Z \wedge^{L} X\right)_{n}=\underset{W \subset \mathcal{U}}{\operatorname{colim}} \operatorname{Map}_{*}\left(\left|S^{W-\mathbb{R}^{n}}\right|,|Z| \wedge\left|X\left(S^{W}\right)\right|\right) .
$$

Here $\mathcal{U}$ denotes a complete $S^{1}$-universe (e.g. $\left.\mathcal{U}=\bigoplus_{n \in \mathbb{Z}, \alpha \in \mathbb{N}} \mathbb{C}(n)_{\alpha}\right)$ and the colimit runs over finite dimensional sub inner spaces $W$ of $\mathcal{U}$ containing $\mathbb{R}^{n}$. The symbol $W-\mathbb{R}^{n}$ denotes the orthogonal complement of $\mathbb{R}^{n}$ in $W$ and $S^{W}$ denotes the singular complex of the one point compactification of $W$. There are several possible actions of $S^{1}$ on $\left|X\left(S^{W}\right)\right|$. Using the functoriality of $X$ the action of $S^{1}$ on $S^{W}$ induces an action of $S^{1}$ on $X\left(S^{W}\right)$. On the other hand forgetting the action of $S^{1}$ on $S^{W}$ we still have a cyclic structure on $X\left(S^{W}\right)$ given rise to an action of $S^{1}$ on $\left|X\left(S^{W}\right)\right|$. The two actions just described commute and therefore we end up with an action of $S^{1} \times S^{1}$ on $\left|X\left(S^{W}\right)\right|$. We shall always let $S^{1}$ act on $\left|X\left(S^{W}\right)\right|$ by pulling back the action of $S^{1} \times S^{1}$ along the diagonal $S^{1} \rightarrow S^{1} \times S^{1}$. Letting $S^{1}$ act on the pointed mapping space $\operatorname{Map}_{*}\left(\left|S^{W-\mathbb{R}^{n}},\right| Z|\wedge| X\left(S^{W}\right) \mid\right)$ by conjugation we obtain an action of $S^{1}$ on $\left(Z \wedge^{L} X\right)_{n}$ and we obtain a spectrum $\left(Z \wedge^{L} X\right)^{H}$ with $\left(Z \wedge^{L} X\right)_{n}^{H}=\left(\left(Z \wedge^{L} X\right)_{n}\right)^{H}$ for every closed subgroup $H$ of $S^{1}$. Since the map $U \wedge X(V) \rightarrow X(U \wedge V)$ is a cofibration for every $U$ and $V$ the above construction is homotopically meaningful. (See the discussion in [13, Appendix A].)

In particular we can consider the spectrum $\left(S^{0} \wedge^{L} X\right)^{H}$. There is a map $X^{H} \rightarrow$ $\left(S^{0} \wedge^{L} X\right)^{H}$ induced by the map $\left|X\left(S^{n}\right)\right| \rightarrow \operatorname{Map}_{*}\left(\left|S^{W}\right|,\left|X\left(S^{n} \wedge S^{W}\right)\right|\right)$. According to [13, prop. 2.4] this map is an equivalence when $X=\operatorname{THH}(L)$ and $H$ is finite.

Using the standard cyclic model of $S^{1}$ we can consider $E S^{1}$ as a cyclic space, and we can consider the spectrum $\left(E S_{+}^{1} \wedge^{L} X\right)^{C}$. According to [14, thm. 7.1. p. 97] it represents the $C$-homotopy orbit spectrum $X_{h C}$ of $X$ in the homotopy category when $C$ is finite and $\left(E S_{+}^{1} \wedge^{L} X\right)^{S^{1}}$ represents the suspension 
$S^{1} \wedge X_{h S^{1}}$ of the $S^{1}$-homotopy orbits of $X$. From now on we shall always use these representatives for homotopy orbits. The inclusions of fixed points $\left(E S_{+}^{1} \wedge^{L} X\right)^{C_{a q}} \rightarrow\left(E S_{+}^{1} \wedge^{L} X\right)^{C_{a}}$ and $\left(E S_{+}^{1} \wedge^{L} X\right)^{S^{1}} \rightarrow\left(E S_{+}^{1} \wedge^{L} X\right)^{C_{a}}$ represent the transfer maps $\operatorname{trf}_{q}: X_{h C_{q a}} \rightarrow X_{h C_{a}}$ and $\operatorname{trf}_{\infty}: S^{1} \wedge X_{h S^{1}} \rightarrow X_{h C_{a}}$ respectively. We shall always use these representatives for the the transfer maps.

Definition 4.4 A $p$-cyclotomic Gamma space is a Gamma epicyclic space $X$ satisfying the following two conditions.

(1) The map $X^{C} \rightarrow\left(S^{0} \wedge^{L} X\right)^{C}$ is an equivalence for every finite $p$-subgroup $C$ of $S^{1}$.

(2) The norm map $N: X_{h C_{p^{n}}} \rightarrow X^{C_{p^{n}}}$ defined as the composite

$$
X_{h C_{p^{n}}}=\left(E S_{+}^{1} \wedge^{L} X\right)^{C_{p^{n}}} \rightarrow\left(S^{0} \wedge^{L} X\right)^{C_{p^{n}}} \simeq X^{C_{p^{n}}}
$$

fits into a cofibration sequence $X_{h C_{p^{n}}} \stackrel{N}{\rightarrow} X^{C_{p^{n}}} \stackrel{R_{p}}{\rightarrow} X^{C_{p^{n-1}}}$ for every $n \geq 1$.

Note that the norm map is only defined in the homotopy category and that the diagram

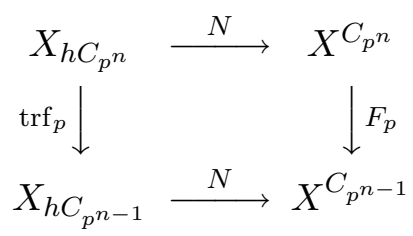

commutes. It is proven in [13, lemma 2.5] and [13, prop. 2.4] that $\operatorname{THH}(L)$ satisfies (1) and (2) above. In conclusion $\mathrm{THH}(L)$ is a $p$-cyclotomic Gamma space.

Below we shall use the following lemma due to Goodwillie. It can be found in [17] as lemma 4.4.9.

Lemma 4.5 For any epicyclic Gamma space $X$ the $S^{1}$-transfer induces a map

$$
S^{1} \wedge X_{h S^{1}} \rightarrow \operatorname{holim}_{\operatorname{trf}_{p}} X_{h C_{p^{n}}} .
$$

This map becomes an equivalence after $p$-completion.

Let us sketch an alternative proof of this lemma. Since $\left(E S_{+}^{1}\right)_{k}=\left(S_{+}^{1}\right)^{\wedge k+1}$ it suffices to show that the map $\left(\left(S_{+}^{1}\right)^{\wedge k+1} \wedge^{L} X\right)^{S^{1}} \rightarrow \underset{F_{p}}{\operatorname{holim}}\left(\left(S_{+}^{1}\right)^{\wedge k+1} \wedge^{L} X\right)^{C_{p^{n}}}$ is an equivalence for every $k \geq 0$. There is an isomorphism $\left(S_{+}^{1}\right)^{\wedge k+1} \wedge{ }^{L} X \cong$ 
$S_{+}^{1} \wedge^{L}\left(\left(S_{+}^{1}\right)^{\wedge k} \wedge X\right)$, where $\left(S_{+}^{1}\right)^{\wedge k} \wedge X$ denotes the Gamma cyclic space $n^{+} \mapsto$ $\left(S_{+}^{1}\right)^{\wedge k} \wedge X\left(n^{+}\right)$. Therefore the proof of lemma 4.5 reduces to showing that the map $\left(S_{+}^{1} \wedge^{L} X\right)^{S^{1}} \rightarrow \underset{F_{p}}{\operatorname{holim}}\left(S_{+}^{1} \wedge^{L} X\right)^{C_{p^{n}}}$ becomes an equivalence after completion at $p$. This is the statement of [13, lemma 8.2].

\subsection{Cyclotomically filtered Gamma spaces}

In this section we shall present a filtered version of $p$-cyclotomic Gamma spaces. Let us begin with a filtered version of the notion of an epicyclic space.

Definition 4.6 An epicyclic filtered space is a filtered cyclic space $Y$ equipped with maps $r_{q}: Y_{q j-1}(s)^{C_{q}} \rightarrow Y_{j-1}([s / q])$ for all $q, j \geq 1$ and $s \in \mathbb{Z}$, satisfying:

(1) $r_{q}:\left(\operatorname{sd}_{q} Y\right)(s)^{C_{q}} \rightarrow Y([s / q])$ is cyclic.

(2) $r_{a} \circ r_{q}=r_{a q}:\left(\operatorname{sd}_{a q} Y\right)(s)^{C_{a q}} \rightarrow Y([s /(a q)])$.

(3) $r_{1}$ is the identity.

Here $[s / q]$ denotes the greatest integer $\leq s / q$. Write $Y^{C_{a}}(s)$ for the topological space $\left|\left(\operatorname{sd}_{a} Y(s)\right)^{C_{a}}\right| \cong|Y(s)|^{C_{a}}$. There is a Frobenius map $f_{q}: Y^{C_{a q}}(s) \cong$ $|Y(s)|^{C_{a q}} \rightarrow|Y(s)|^{C_{a}} \cong Y^{C_{a}}(s)$ induced by inclusion of fixed points.

A Gamma epicyclic filtered space is a Gamma object in the category of epicyclic filtered spaces.

Topological Hochschild homology of an FSP filtered by cofibrations is the main example of a Gamma epicyclic filtered space. The restriction map

$$
r_{q}: \operatorname{sd}_{q} \operatorname{THH}\left(L, n^{+}\right)(s)^{C_{q}} \rightarrow \operatorname{THH}\left(L, n^{+}\right)([s / q])
$$

is defined degreewise by the following chain of maps:

$$
\begin{aligned}
& \left(\operatorname{sd}_{q} \operatorname{THH}\left(L, n^{+}\right)(s)\right)_{k}^{C_{q}} \cong \\
& \underset{\left(n_{0}, \ldots, n_{k}\right) \in I^{k+1}}{\operatorname{hocolim}} F\left(\left(S^{n_{0}} \wedge \cdots \wedge S^{n_{k}}\right)^{\wedge q},\left(L\left(S^{n_{0}}\right) \wedge \cdots \wedge L\left(S^{n_{k}}\right)\right)^{\wedge q}(s) \wedge n^{+}\right)^{C_{q}} \rightarrow \\
& \left.\underset{\left(n_{0}, \ldots, n_{k}\right) \in I^{k+1}}{\operatorname{hocolim}} F\left(\left(\left(S^{n_{0}} \wedge \cdots \wedge S^{n_{k}}\right)^{\wedge q}\right)^{C_{q}},\left(L\left(S^{n_{0}}\right) \wedge \cdots \wedge L\left(S^{n_{k}}\right)\right)^{\wedge q}(s) \wedge n^{+}\right)^{C_{q}}\right) \\
& \cong \underset{\left(n_{0}, \ldots, n_{k}\right) \in I^{k+1}}{\operatorname{hocolim}} F\left(S^{n_{0}} \wedge \cdots \wedge S^{n_{k}},\left(L\left(S^{n_{0}}\right) \wedge \cdots \wedge L\left(S^{n_{k}}\right)\right)([s / q]) \wedge n^{+}\right)= \\
& \operatorname{THH}\left(L, n^{+}\right)([s / q])_{k} \text {. }
\end{aligned}
$$

The first isomorphism is due to the isomorphism $\left.\underset{I^{q(k+1)}}{\operatorname{hocolim}} Z\right)^{C_{q}} \cong \underset{I^{k+1}}{\operatorname{hocolim}} Z^{C_{q}}$. The second map is given by restriction to fixed points and the last isomorphism 
is induced by the point set isomorphism $\left(X^{\wedge q}\right)(s)^{C_{q}} \cong X([s / q])$ for $X$ a space filtered by cofibrations. This last isomorphism is not obvious though, so we state it as a lemma.

Lemma 4.7 Let $Y$ be a filtered space, filtered by cofibrations. There is an isomorphism $\left(Y^{\wedge q}(s)\right)^{C_{q}} \cong Y([s / q])$

Proof The diagonal induces a map

$$
Y([s / q]) \stackrel{\cong}{\rightrightarrows}\left(\left(Y^{\wedge q}\right)(s)\right)^{C_{q}} .
$$

We will show that this is an isomorphism of simplicial sets. We may assume that $Y$ is a discrete set filtered by injections. We note that by the pushout diagram in the proof of lemma 2.3 the map $\left(Y^{\wedge q}\right)(i) \rightarrow\left(Y^{\wedge q}\right)(i+1)$ is an injection for all $i \in \mathbb{Z}$, and therefore we have an injection

$$
Y^{\wedge q}(s) \hookrightarrow Y^{\wedge q}(\infty) \cong(Y(\infty))^{\wedge q},
$$

with the convention that $Y^{\wedge q}(\infty)=\operatorname{colim}_{i} Y^{\wedge q}(i)$ and $Y(\infty)=\operatorname{colim}_{i} Y(i)$. There is a commutative diagram

$$
\begin{array}{ccc}
Y([s / q]) & \rightarrow & \left(Y^{\wedge q}(s)\right)^{C_{q}} \\
\downarrow & & \downarrow \\
Y(\infty) & \rightarrow & \left(Y(\infty)^{\wedge q}\right)^{C_{q}}
\end{array}
$$

where the vertical arrows are injections. It follows from the diagram that the map $Y([s / q]) \rightarrow\left(Y^{\wedge q}(s)\right)^{C_{q}}$ is injective. To see that it is onto, let us pick a representative $\left(\left(a_{1}, \ldots, a_{q}\right),\left(y_{1}, \ldots, y_{q}\right)\right)$ for a point $y$ in

$$
\left(Y^{\wedge q}(s)\right)=\underset{a_{1}+\cdots+a_{q} \leq s}{\operatorname{colim}} Y\left(a_{1}\right) \wedge \cdots \wedge Y\left(a_{q}\right),
$$

fixed under the $C_{q}$-action. From the condition $a_{1}+\cdots+a_{q} \leq s$, it follows that there exists an $i$ such that $a_{i} \leq s / q$. Since the image of $y$ in $Y(\infty)^{\wedge q}$ is a fixed point, we must have that $\left(a_{1}, y_{1}\right), \ldots,\left(a_{q}, y_{q}\right)$ represent the same element in $Y(\infty)$. Since the map $Y^{\wedge q}(s) \rightarrow Y^{\wedge q}(\infty)$ is injective, it follows that $\left(\left(a_{i}, \ldots, a_{i}\right),\left(y_{i}, \ldots, y_{i}\right)\right)$ represents $y$, and we can conclude that the map $Y([s / q]) \rightarrow\left(Y^{\wedge q}(s)\right)^{C_{q}}$ is onto.

Definition 4.8 A p-cyclotomic filtered Gamma space is a Gamma epicyclic filtered space $X$ satisfying the following two conditions.

(1) The map $X^{C}(s) \rightarrow\left(S^{0} \wedge^{L} X(s)\right)^{C}$ is an equivalence for every finite $p$ subgroup $C$ of $S^{1}$ and $s \in \mathbb{Z}$. 
(2) The norm map $N: X(s)_{h C_{p^{n}}} \rightarrow X^{C_{p^{n}}}(s)$ defined as the composite

$$
X(s)_{h C_{p^{n}}}=\left(E S_{+}^{1} \wedge^{L} X(s)\right)^{C_{p^{n}}} \rightarrow\left(S^{0} \wedge^{L} X(s)\right)^{C_{p^{n}}} \simeq X^{C_{p^{n}}}(s)
$$

fits into a cofibration sequence $X(s)_{h C_{p^{n}}} \stackrel{N}{\rightarrow} X^{C_{p^{n}}}(s) \stackrel{R_{p}}{\rightarrow} X^{C_{p^{n-1}}}([s / p])$ for every $n \geq 1$.

The proof of [13, prop 2.4] shows that for any filtered FSP $L$ the Gamma cyclic space $\operatorname{THH}(L)(s)$ satisfies (1) above. If $L$ is filtered by cofibrations we have by lemma 4.7 that

$$
\left(\left(L\left(S^{i_{0}}\right) \wedge \cdots \wedge L\left(S^{i_{k}}\right)\right)^{\wedge r}(s)\right)^{C_{q}} \cong\left(L\left(S^{i_{0}}\right) \wedge \cdots \wedge L\left(S^{i_{k}}\right)\right)^{\wedge r / q}([s / q]) .
$$

It follows from lemma 3.7 that its connectivity is at least $\left(i_{0}+\cdots+i_{k}\right) r / q-1$. The proof of [13, prop 2.5] together with the above observation shows that $\operatorname{THH}(L)$ satisfies $(2)$ in the above definition. Hence $\operatorname{THH}(L)$ is a $p$-cyclotomic filtered Gamma space.

\section{$5 \quad$ Filtered topological cyclic homology}

Given a Gamma epicyclic filtered space $X$, the Gamma spaces $X(-1), X(0)$ and $X(\infty)=\operatorname{colim}_{s} X(s)$ come equipped with an epicyclic structure. In this section we shall define a filtered Gamma space $T C=T C(X, p)$, the topological cyclic homology of $X$. This will be a generalization of non-filtered topological cyclic homology in the sense that $T C(s)$ is the (non-filtered) topological cyclic homology $T C(X(s), p)$ of the Gamma epicyclic space $X(s)$ for $s=-1,0, \infty$. Here we use the notation $T C(\infty)=\operatorname{colim} T C(s)$. The sign of $s$ plays an important role in the definition of $T C(s)$. In fact $T C(s)$ is defined using only the $X(r)$ where $r$ has the same sign as $s$.

We start by defining $t c(Y)(s)$ for a filtered epicyclic space $Y$. Let us start by defining $t c(Y)(s)$ when $s \leq 0$. In this case $s \leq[s / p]$. (Recall that $[s / p]$ is the greatest integer less that or equal to $s / p$.) Therefore there is an inclusion $Y(s) \stackrel{i}{\rightarrow} Y([s / p])$. Using this inclusion and the restriction map $Y(s)^{C_{p^{n}}} \stackrel{r_{p}}{\rightarrow}$ $Y([s / p])^{C} p^{n-1}$ we obtain maps

$$
\prod_{n \geq 0} Y(s)^{C_{p^{n}}} \underset{i(s)}{\stackrel{r(s)}{\rightrightarrows}} \prod_{n \geq 0} Y([s / p])^{C_{p^{n}}}
$$


Let us define $\operatorname{tr}(Y)(s)$ to be the homotopy equalizer of $r(s)$ and $i(s)$. The Frobenius maps $Y(s)^{C_{p^{n}}} \stackrel{f(s)}{\longrightarrow} Y(s)^{C_{p^{n-1}}}$ commute with $r(s)$ and $i(s)$, and therefore they induce an endomorphism $f(s)$ of $\operatorname{tr}(Y)(s)$. We define $\operatorname{tc}(Y)(s)$ to be the homotopy equalizer of $f(s)$ and the identity. When $s=-1,0$ this definition of $t c(Y)(s)$ agrees with the definition of $t c(Y(s))$ given in section 4.1. There is an alternative definition of $t c(Y)(s)$ where we interchange the roles of $r$ and $f$ going as follows: We let $t f(Y)(s)$ denote the homotopy equalizer of the maps

$$
\prod_{n \geq 0} Y(s)^{C_{p^{n}}} \stackrel{\stackrel{f(s)}{\rightarrow}}{\underset{\mathrm{id}}{\rightrightarrows}} \prod_{n \geq 0} Y(s)^{C_{p^{n}}} .
$$

$t c(Y)(s)$ is equivalent to the homotopy equalizer of the maps $r(s)$ and $i(s)$ from $t f(Y)(s)$ to $t f(Y)([s / p])$.

Now let us define $\operatorname{tc}(Y)(s)$ when $s \geq 0$. The restriction map induces an endomorphism $r(s)$ on the product $\prod_{n \geq 0} Y\left(s p^{n}\right)^{C_{p^{n}}}$. We define $\operatorname{tr}(Y)(s)$ to be the homotopy equalizer of $r(s)$ and the identity. The Frobenius map induces a map $f(s): \operatorname{tr}(Y)(s) \rightarrow \operatorname{tr}(Y)(s p)$. Since $s \leq s p$, there is an inclusion $Y(s) \rightarrow Y(s p)$ inducing a map $i(s): \operatorname{tr}(Y)(s) \rightarrow \operatorname{tr}(Y)(s p)$. We define $\operatorname{tc}(Y)(s)$ to be the homotopy equalizer of $f(s)$ and $i(s)$. When $s=0$ this definition of $t c(Y)(s)$ agrees with the definition given above.

Given a Gamma epicyclic filtered space $X$ we have spaces $t c\left(X\left(S^{n}\right)\right)(s)$. Filtered topological cyclic homology at the prime $p$ of $X$ is the spectrum $T C=$ $T C(X, p)$ with $T C(X, p)(s)_{n}=t c\left(X\left(S^{n}\right), p\right)(s)$. Similarly let $T R=T R(X, p)$ be the filtered spectrum with $T R(X, p)(s)_{n}=\operatorname{tr}\left(X\left(S^{n}\right), p\right)(s)$. Let us note that $T C(X(s), p) \cong T C(X, p)(s)$ for $s=-1,0, \infty$. This fact together with the two following lemmas is our justification for the definition of $T C(X, p)$. For $s=-1,0, \infty$ the spectrum $\operatorname{TR}(X(s), p)$ can be rewritten as the sequential homotopy limit of $X(s)^{C_{p}}$ with respect to the restriction maps. There is no such rewriting possible for $s \neq-1,0, \infty$.

Lemma 5.1 Let $X$ be a $p$-cyclotomic filtered Gamma space filtered by cofibrations. Suppose that the connectivity of the map $X(s) \rightarrow X(\infty)$ tends to infinity as $s$ grows. Then $X(\infty)$ is a $p$-cyclotomic Gamma space and $T C(X, p)(\infty)$ is stably equivalent to $T C(X(\infty), p)$.

Recall that $X(\infty)$ is the Gamma epicyclic space space with underlying Gamma space $\operatorname{colim}_{s} X(s)$, and that $T C(\infty)=\operatorname{colim}_{s} T C(s)$. 
Proof Suppose that the map $X(s) \rightarrow X(\infty)$ is $k$-connected for $s \geq N \geq 0$. Using the cofibration sequence

$$
X\left(s p^{n}\right)_{h C_{p^{n}}} \rightarrow X\left(s p^{n}\right)^{C_{p^{n}}} \rightarrow X\left(s p^{n-1}\right)^{C_{p^{n-1}}}
$$

we can by induction show that the map $X\left(s p^{n}\right)^{C_{p^{n}}} \rightarrow X(\infty)^{C_{p^{n}}}$ is $k$-connected when $s \geq N$, for all $n$. It follows that the map

$$
\prod_{n \geq 0} X\left(s p^{n}\right)^{C_{p^{n}}} \rightarrow \prod_{n \geq 0} X(\infty)^{C_{p^{n}}}
$$

is $k$-connected when $s \geq N$. Therefore the map $T R(s) \rightarrow T R(X(\infty), p)$ is at least $k-1$-connected, and the map $T C(s) \rightarrow T C(X(\infty), p)$ is at least $k-2$ connected when $s \geq N$.

The next lemma says that if $X$ is a $p$-cyclotomic filtered Gamma space and the connectivity of $X(s)$ tends to infinity as $s$ decreases then $\underset{s}{\operatorname{holim}} T C(s)$ is contractible.

Lemma 5.2 Let $X$ be a $p$-cyclotomic filtered Gamma space. If $s \leq 0$ then $T R(s)$ and $\Sigma T C(s)$ are at least as highly connected as $X(s)$.

Proof Let $T R^{m}(s)$ denote the homotopy equalizer of the diagram

$$
\prod_{0 \leq n \leq m} X(s)^{C_{p^{n}}} \underset{I(s)}{\stackrel{R(s)}{\rightrightarrows}} \prod_{0 \leq n \leq m-1} X([s / p])^{C_{p^{n}}},
$$

where $I(s)$ forgets the $m$ 'th coordinate, and otherwise $I(s)$ and $R(s)$ are truncations of the maps defining $T R(s)$. There is an obvious map $T R^{m}(s) \rightarrow$ $T R^{m-1}(s)$ induced by projection away from the last factors of the products. Using the norm cofibration sequence the fibre of this map may be identified with $X(s)_{h C_{p} m}$. Since homotopy limits commute we have that $T R(s)$ is the homotopy limit of the sequence

$$
\cdots \rightarrow T R^{m}(s) \rightarrow T R^{m-1}(s) \rightarrow \cdots \rightarrow T R^{0}(s) .
$$

Since homotopy orbits preserve connectivity and $T R^{0}(s)=X(s), T R(s)$ is a sequential homotopy limit of spaces as least as connected as $X(s)$ and with homotopy fibres as least as connected as $X(s)$. It follows that $T R(s)$ is at least as highly connected as $X(s)$. Since homotopy equalizers at most lower connectivity by one we have that $\Sigma T C(s)$ is at least as highly connected as $T R(s)$. 
Given a map $A \rightarrow B$ of spectra we shall denote the homotopy cofibre by $B / A$.

Lemma 5.3 Let $X$ be a $p$-cyclotomic filtered Gamma space, let $s<0$, and assume that $p s \leq t<s$. After $p$-completion $T C(s) / T C(t)$ is equivalent to $S^{1} \wedge(X(s) / X(t))_{h S^{1}}$.

Proof Since $s \leq[t / p]$, the inclusion $X(s) \rightarrow X([s / p])$ induces the trivial map from $X(s) / X(t)$ to $X([s / p]) / X([t / p])$. Therefore the homotopy equalizer of the maps induced by $I(s)$ and $R(s)$ on the quotients of the products in the definition of $T R$ agrees with the homotopy fibre of the map induced by $R(s)$. Using the norm cofibration sequence we can identify this fibre with $\prod_{n \geq 0}(X(s) / X(t))_{h C_{p^{n}}}$. Since $F_{p} N=N \operatorname{trf}_{p}$, where $N: X(s)_{h C_{p^{n}}} \rightarrow X(s)^{C_{p^{n}}}$ denotes the norm map, and where $\operatorname{trf}_{p}$ denotes the transfer map, we have that $T C(s) / T C(t) \simeq \operatorname{holim}_{\operatorname{trf}_{p}}(X(s) / X(t))_{h C_{p^{n}}}$. The lemma now follows from lemma 4.5 .

Note that the above lemma applies to the filtration quotients $T C(s) / T C(s-1)$. For our main theorem the case $s=-1$ and $t=-p$ is of particular interest. In that case we get by lemma 5.2 that if $X(-p)$ is $k$-connected, then after $p$-adic completion the map

$$
T C(-1) \rightarrow \frac{T C(-1)}{T C(-p)} \simeq S^{1} \wedge\left(\frac{X(-1)}{X(-p)}\right)_{h S^{1}}
$$

is $k$-connected.

It follows from remark 3.11 that for an FSP $L$ filtered by cofibrations and with $L(s)=L(0)$ for $s \geq 0$ we have that $T C(0) / T C(-1)$ is isomorphic to $T C(L(0) / L(-1), p)$.

\section{Relative $K$-theory of nilpotent ideals}

In this section we shall prove the following theorem relating relative $K$-theory and relative cyclic Homology. One good reference for cyclic homology is the book of Loday [15].

Theorem 6.1 Let $R$ be a simplicial ring with an ideal $I$ satisfying $I^{m}=$ 0 . Suppose that $R$ and $R / I$ are flat as modules over $\mathbb{Z}$. Then there is an isomorphism of homotopy groups of $p$-adic completions

$$
\pi_{i} K(R, I)_{p}^{\wedge} \cong \pi_{i-1} H C(R, I)_{p}^{\wedge}
$$


when $0 \leq i<p /(m-1)-2$ and a surjection

$$
\pi_{i} K(R, I)_{p}^{\wedge} \rightarrow \pi_{i-1} H C(R, I)_{p}^{\wedge}
$$

when $i<p /(m-1)-1$.

Recall that $K(R, I)$ is the homotopy fibre of the map $K(R) \rightarrow K(R / I)$ and that $H C(R, I)$ is the homotopy fibre of the map $H C(R) \rightarrow H C(R / I)$.

The proof uses the results of the previous section plus a number of results about $T C$ proven elsewhere. We shall collect the statements of these results for the convenience of the reader. The following result is due to McCarthy [19].

Theorem 6.2 Suppose $f: R \rightarrow S$ is a homomorphism of simplicial rings and that $\pi_{0}(f)$ is surjective and has nilpotent kernel. Then the diagram

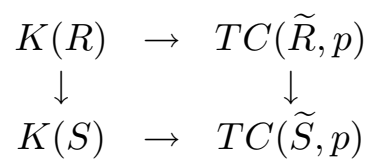

is homotopy Cartesian after p-adic completion.

Suppose that $f$ in the above theorem is degreewise surjective and let $I \subseteq R$ denote its kernel. Let $T C(\widetilde{R}, \widetilde{I}, p)$ denote the homotopy fibre of the map $T C(\widetilde{R}, p) \rightarrow T C(\widetilde{S}, p)$. Then the theorem says that the map $K(R, I) \rightarrow$ $T C(\widetilde{R}, \widetilde{I}, p)$ is an equivalence after $p$-adic completion. The theorem in particular applies in the situation where $I$ is a nilpotent ideal in $R$.

Lemma 6.3 Let $R$ be a ring which is flat as a module over $\mathbb{Z}$. Then the map $\pi_{i} \operatorname{THH}(\widetilde{R})_{p}^{\wedge} \rightarrow \pi_{i} \operatorname{HH}(R)_{p}^{\wedge}$ is an isomorphism when $i \leq 2 p-2$.

Proof In [20, thm 4.1] Pirashvili and Waldhausen have established a spectral sequence with $E^{2}$-term $E_{s, t}^{2}=\operatorname{HH}_{s}\left(R, \pi_{t} \mathrm{THH}(\widetilde{\mathbb{Z}}, \widetilde{R})\right)$ converging towards $\pi_{s+t} \operatorname{THH}(\widetilde{R})$. The lemma follows from the fact that $\pi_{0} \operatorname{THH}(\widetilde{\mathbb{Z}}, \widetilde{R})=R$ and that $\pi_{i} \operatorname{THH}(\widetilde{\mathbb{Z}}, \widetilde{R})_{p}^{\wedge}=0$ when $1 \leq i \leq 2 p-2$.

The following result is dual to a result of Cohen and Jones [9, lemma 1.3]. We give an alternative proof inspired by a more elementary proof due to Bökstedt.

Proposition 6.4 Let $A$ be a cyclic object in the category of abelian groups, and let $\widetilde{A}$ denote the Gamma cyclic space with $\widetilde{A}\left(n^{+}\right)=A \otimes_{\mathbb{Z}} \widetilde{\mathbb{Z}}\left(n^{+}\right)$. Then there is a natural isomorphism $H C_{*}(A) \cong \pi_{*}\left(\widetilde{A}_{h S^{1}}\right)$. 
Recall that $\widetilde{A}_{h S^{1}}$ is the $S^{1}$ homotopy orbit spectrum associated to the spectrum $n \mapsto\left|\widetilde{A}\left(S^{n}\right)\right|$.

Proof We refer to [15, section 6.2] for the notation used in this proof. It is well known (see e.g. [15, theorem 6.2.8], or use the argument below) that there is an isomorphism $H C_{*}(A) \cong \operatorname{Tor}_{*}^{\Lambda^{\text {op }}}(\mathbb{Z}, A)$. To see that $\pi_{*}\left(\widetilde{A}_{h S^{1}}\right)$ also is isomorphic to this Tor group, it suffices to check the usual properties determining Tor groups up to isomorphism (see e.g. [8, theorem V.6.1]). Firstly we note that there are isomorphisms $\pi_{0}\left(\widetilde{A}_{h S^{1}}\right) \cong \pi_{0}(|A|) \cong \mathbb{Z} \otimes_{\Lambda^{\text {op }}} A$. Secondly the representable functors $\mathbb{Z} \Lambda^{n}$ with $\mathbb{Z} \Lambda^{n}([m])=\mathbb{Z}[\Lambda([m],[n])]$ form a set of projective generators for the category of cyclic objects in the category of abelian groups, that is, every projective object in this category is a quotient of a sum of objects of the form $\mathbb{Z} \Lambda^{n}$. Since $\left|\mathbb{Z} \Lambda^{n}\right| \cong\left|\mathbb{Z}\left[S^{1} \times \Delta^{n}\right]\right|$ we can use lemma 3.2 to see that $\widetilde{\mathbb{Z} \Lambda^{n}}{ }_{h S^{1}} \simeq \widetilde{\mathbb{Z}}$, and hence $\pi_{i}\left(\widetilde{\mathbb{Z} \Lambda^{n}} h S^{1}\right)=0$ for $i>0$. Thirdly, given a short exact sequence $A^{\prime} \rightarrow A \rightarrow A^{\prime \prime}$ of cyclic objects in the category of abelian groups, we obtain a cofibration sequence $\widetilde{A}^{\prime} \rightarrow \widetilde{A} \rightarrow \widetilde{A}^{\prime \prime}$ of spectra with an action of $S^{1}$. Since homotopy orbits take cofibration sequences to cofibration sequences we obtain a long exact sequence of homotopy groups of homotopy orbits.

The proposition in particular says that there is an isomorphism $\left.\pi_{i} \widetilde{\mathrm{HH}(R)}\right)_{h S^{1}} \cong$ $H C_{i}(R)$. To prove theorem 6.1 we also need the following lemma.

Lemma 6.5 Let $L$ be an FSP filtered by cofibrations. Suppose that $L(s)=$ $L(0)$ for $s \geq 0$, and that there exists $m \geq 0$ such that $L(-m)=*$. Then $\pi_{k} \operatorname{THH}(L)(s)=0$ when $k<-s /(m-1)-1$.

Proof Recall that $\left(L\left(S^{n_{0}}\right) \wedge \cdots \wedge L\left(S^{n_{k}}\right)\right)(s)$ is the colimit running over $i_{0}+$ $\cdots+i_{k} \leq s$ of

$$
L\left(S^{n_{0}}\right)\left(i_{0}\right) \wedge \cdots \wedge L\left(S^{n_{k}}\right)\left(i_{k}\right) .
$$

If $i_{0}+\cdots+i_{k} \leq s$ then there exists an $\alpha$ such that $i_{\alpha} \leq s /(k+1)$. Therefore the smash product is zero if $s /(k+1)<-m+1$, or equivalently if $k<$ $-s /(m-1)-1$, and hence $\operatorname{THH}(L)(s)_{k}=0$ if $k<-s /(m-1)-1$.

Using the above lemma and lemma 5.3, or rather the remark after it, we obtain the following.

Proposition 6.6 Let $L$ be an FSP filtered by cofibrations, and suppose that $L(s)=L(0)$ for $s \geq 0$, and that there exists $m \geq 0$ such that $L(X)(-m)=*$ 
for all $X$. Let $T C=T C(L, p)$, and $\mathrm{THH}=\mathrm{THH}(L)$. After completion at $p$ there are maps

$$
T C(-1) \rightarrow \frac{T C(-1)}{T C(-p)} \simeq S^{1} \wedge\left(\frac{\mathrm{THH}(-1)}{\mathrm{THH}(-p)}\right)_{h S^{1}} \leftarrow S^{1} \wedge \mathrm{THH}(-1)_{h S^{1}} .
$$

Here $\operatorname{THH}(-p)$ is $p /(m-1)-2$-connected and $T C(-p)$ is $p /(m-1)-3$ connected, and therefore the map pointing to the right is $p /(m-1)-2$-connected and the map pointing to the left is $p /(m-1)-3$-connected.

Now let $R$ denote a ring with an ideal $I$ satisfying that $I^{m}=0$. Considering the $I$-adic filtration $0=I^{m} \subseteq \cdots \subseteq I \subseteq R$ of $R$ we obtain a filtered ring $R$ with $R(s)=I^{-s}$ for $s<0$ and with $R(s)=R$ for $s \geq 0$. By the construction in 3.6 we obtain a filtered FSP $\widetilde{R}$ with $\widetilde{R}\left(n^{+}, s\right)=\widetilde{\mathbb{Z}}\left(n^{+}\right) \otimes_{\mathbb{Z}} R(s)$. As remarked after $3.10 \mathrm{THH}(R)(-1)$ is equivalent to the homotopy fibre of the map $\operatorname{THH}(R) \rightarrow$ $\operatorname{THH}(R / I)$. Since homotopy limits commute we have that

$$
T C(R, I, p)=T C(R, p)(-1) .
$$

Applying McCarthy's theorem 6.2 and the above proposition we obtain an isomorphism

$$
\pi_{i} K(R, I)_{p}^{\wedge} \cong \pi_{i-1}\left(\mathrm{THH}(R)(-1)_{h S^{1}}\right)_{p}^{\wedge}
$$

when $i<p /(m-1)-2$ and we have a surjection

$$
\pi_{i} K(R, I)_{p}^{\wedge} \rightarrow \pi_{i-1}\left(\operatorname{THH}(R)(-1)_{h S^{1}}\right)_{p}^{\wedge}
$$

when $i<p /(m-1)-1$. Using lemma 6.4 and lemma 6.3 we can complete the proof of theorem 6.1 .

Proof of theorem 6.1 Let us write $\operatorname{THH}(R, I)$ instead of $\operatorname{THH}(R)(-1)$. We have an isomorphism $\pi_{i} K(R, I)_{p}^{\wedge} \cong \pi_{i-1}\left(\operatorname{THH}(R, I)_{h S^{1}}\right)_{p}^{\wedge}$ when $i<p /(m-1)-$ 2 and a surjection $\pi_{i} K(R, I)_{p}^{\wedge} \rightarrow \pi_{i-1}\left(\operatorname{THH}(R, I)_{h S^{1}}\right)_{p}^{\wedge}$ when $i<p /(m-1)-1$. By lemma 6.3 there is for $i \leq 2 p-1$ an isomorphism $\pi_{i-1}\left(\operatorname{THH}(R, I)_{h S^{1}}\right)_{p}^{\wedge} \cong$ $\pi_{i-1}\left(\mathrm{HH}(R, I)_{h S^{1}}\right)_{p}^{\wedge}$. Splicing these maps with the isomorphism of lemma 6.4 we obtain the asserted isomorphism $\pi_{i} K(R, I)_{p}^{\wedge} \cong \pi_{i-1} H C(R, I)_{p}^{\wedge}$ when $i<$ $p /(m-1)-2$ and the surjection $\pi_{i} K(R, I)_{p}^{\wedge} \rightarrow \pi_{i-1} H C(R, I)_{p}^{\wedge}$ when $i<$ $p /(m-1)-2$.

\section{Computations in cyclic homology}

In this section we shall compute some derived cyclic homology groups of the $\operatorname{ring} \mathbb{Z} / p^{n}$. The definition of derived cyclic homology depends on the following lemma. A proof can for example be found in [7]. 
Lemma 7.1 Let $A$ be a simplicial ring. There exists a weak equivalence $R \stackrel{\simeq}{\rightrightarrows} A$ of simplicial rings, where $R$ is degreewise free as an abelian group. If $R^{\prime}$ is another ring with underlying degreewise free abelian group, and with a weak equivalence $R^{\prime} \stackrel{\simeq}{\rightrightarrows} A$, then there is a chain of weak equivalences between $R$ and $R^{\prime}$ through simplicial rings with underlying degreewise free abelian groups.

Let $A$ be a simplicial ring, and choose a weak equivalence $R \stackrel{\simeq}{\rightrightarrows} A$ as in the above lemma. That is, with $R$ degreewise free as an abelian group. By functoriality of the Hochschild construction there is a map $\mathrm{HH}(R) \rightarrow \mathrm{HH}(A)$. By definition $\mathrm{HH}(R)$ is the derived Hochschild homology of $A$. (Some authors call it Shukla homology.) By the above lemma it is unique up to weak equivalence. We shall call $H C(R)$ the derived cyclic homology of $A$. We shall use the notation $\widetilde{H C}(A)$ for $H C(R)$.

Given a discrete ring $A$, we can consider it as a constant simplicial ring. This way we obtain derived cyclic homology of discrete rings.

Proposition 7.2 For $0 \leq i<2 p$ the derived cyclic homology of $\mathbb{Z} / p^{n}$ is given as follows:

$$
\widetilde{H C}_{i}\left(\mathbb{Z} / p^{n}\right)= \begin{cases}\mathbb{Z} / p^{n j} & \text { if } i=2(j-1)<2 p \\ 0 & \text { if } i<2 p \text { is odd }\end{cases}
$$

and the relative cyclic homology groups are:

$$
\widetilde{H C}\left(\mathbb{Z} / p^{n}, p^{n-1} \mathbb{Z} / p^{n}\right)= \begin{cases}\mathbb{Z} / p^{j} & \text { if } i=2(j-1)<2 p \\ 0 & \text { if } i<2 p \text { is odd }\end{cases}
$$

Proof Let us consider $\Delta^{1}=\Delta(-,[1])$ as a pointed simplicial monoid as follows. Given $\alpha, \beta:[k] \rightarrow[1]$, we let $(\alpha \cdot \beta)(j)=\alpha(j) \cdot \beta(j)$. The constant map with value $0 \in[1]$ is the base point. There is a pointed submonoid $S^{0}$ of $\Delta^{1}$ consisting of the constant maps. We shall let $R$ denote the subring $\widetilde{\mathbb{Z}}\left(S^{0}\right) \oplus_{p^{n} \widetilde{\mathbb{Z}}\left(S^{0}\right)} p^{n} \widetilde{\mathbb{Z}}\left(\Delta^{1}\right)$ of the pointed monoid ring $\widetilde{\mathbb{Z}}\left(\Delta^{1}\right)$. From the short exact sequence

$$
p^{n} \widetilde{\mathbb{Z}}\left(\Delta^{1}\right) \rightarrow R \rightarrow \mathbb{Z} / p^{n}
$$

it follows that we have a weak equivalence $R \stackrel{\simeq}{\rightarrow} / p^{n}$. The normalized chain complex of $R$ has a generator 1 in degree zero and a generator $t$ in degree 1. The differential takes $t$ to $p^{n} \cdot 1$. The normalized chain complex $C_{*}(R)$ of $\operatorname{HH}(R)$ has a generator of the form $1 \otimes t^{\otimes k}$ in degree $2 k$ and a generator of the form $t^{\otimes k}$ in degree $2 k-1$. The Hochschild boundary $b$ takes $t^{\otimes k}$ to 
$p^{n}\left(1 \otimes t^{\otimes k-1}\right)$. It follows that $\pi_{2 k} \operatorname{HH}(R)=\mathbb{Z} / p^{n}$ and that the odd homotopy groups of $\mathrm{HH}(R)$ are zero. In order to compute cyclic homology of $R$, we need to evaluate the Connes boundary operator $B$ on the chains of the normalized chain complex of $\mathrm{HH}(R)$. The result is that $B\left(t^{\otimes k}\right)=k\left(1 \otimes t^{\otimes k}\right)$, and that $B\left(1 \otimes t^{\otimes k}\right)=0$. It is not easy to compute the higher homology of the bicomplex $(B(R), b, B)$ with $B(R)_{s, t}=C_{t-s}$ and with vertical and horizontal differential induced by $b$ and $B$ respectively. In degrees up to $2 p-1$ the horizontal nonzero differentials become isomorphisms after tensoring with $\mathbb{Z} / p$. Therefore we have that the homology of the total complex of $B(R) \otimes \mathbb{Z} / p$ is a copy of $\mathbb{Z} / p$ in degree $k$ when $0 \leq k \leq 2 p-1$. We can conclude that if $0 \leq i \leq p-1$ then $H C_{2 i}(R)$ is a cyclic $p$-group and $H C_{2 i+1}(R)=0$. To find the order of $H C_{2 i}(R)$ we can consider the spectral sequence associated to the bicomplex $(B(R), b, B)$ with $E^{1}$-term $E_{s, t}^{1}=\mathrm{HH}_{t-s}(R)$. This spectral sequence is concentrated in even total degrees, and therefore there are no nonzero differentials. We know that in degrees up to $2 p-2$ all extensions are maximally nontrivial, and we can read off the stated value of $H C_{i}(R)$.

To see that the map $\widetilde{H C}\left(\mathbb{Z} / p^{n}\right) \rightarrow \widetilde{H C}\left(\mathbb{Z} / p^{n-1}\right)$ is onto when $0 \leq i \leq 2 p-1$ it suffices to check that generators for the group $\mathbb{Z} / p^{(n-1) i} \cong \widetilde{H_{C}}{ }_{2 i}\left(\mathbb{Z} / p^{n-1}\right)$ are in the image. This is easy to see from the induced map of $E^{\infty}$-terms of the spectral sequence considered above.

Lemma 7.3 The map $\pi_{i} T C\left(\mathbb{Z} / p^{n}, p\right) \rightarrow \pi_{i} T C\left(\mathbb{Z} / p^{n-1}, p\right)$ is onto for $1 \leq i \leq$ $p-3$ and $n \geq 2$. Furthermore $\pi_{2 j} T C\left(\mathbb{Z} / p^{n}, p\right)=0$ for $2 \leq 2 j \leq p-3$.

Proof The proof goes by induction on $n$. Suppose that $\pi_{2 j} T C\left(\mathbb{Z} / p^{n-1}, p\right)=0$ for $2 \leq 2 j \leq p-3$. (By the computation of $\pi_{*} T C(\mathbb{Z} / p, p)$ in $[13$, thm. B] this is true for $n=1$.) We have a cofibration sequence

$$
T C\left(\mathbb{Z} / p^{n}, p^{n-1} \mathbb{Z} / p^{n}, p\right) \rightarrow T C\left(\mathbb{Z} / p^{n}, p\right) \rightarrow T C\left(\mathbb{Z} / p^{n-1}, p\right) .
$$

Applying proposition 7.2 , theorem 6.1 with $I=p^{n-1} \mathbb{Z} / p^{n}$ and $m=2$ and theorem 6.2 we find that

$$
\pi_{i} T C\left(\mathbb{Z} / p^{n}, p^{n-1} \mathbb{Z} / p^{n}, p\right) \cong \begin{cases}\mathbb{Z} / p^{j} & \text { when } i=2 j-1 \leq p-3 \\ 0 & \text { when } i \leq p-3 \text { is even. }\end{cases}
$$

The statement of the lemma can be read off from the long exact sequence associated to the cofibration sequence.

Corollary 7.4 For $1 \leq i \leq p-3$, the $K$-groups of $\mathbb{Z} / p^{n}$ are:

$$
\pi_{i} K\left(\mathbb{Z} / p^{n}\right) \cong \begin{cases}0 & \text { if } i \text { is even } \\ \mathbb{Z} / p^{j(n-1)}\left(p^{j}-1\right) & \text { if } i=2 j-1\end{cases}
$$


Proof It follows from the above lemma that the map

$$
\lim _{n} \pi_{i} T C\left(\mathbb{Z} / p^{n}, p\right) \rightarrow \pi_{i} T C\left(\mathbb{Z} / p^{n}, p\right)
$$

is onto and that $\lim _{n}^{1} \pi_{i} T C\left(\mathbb{Z} / p^{n}, p\right)=0$ (see [6] chap IX and XI). We have that holim $T C\left(\mathbb{Z} / p^{n}, p\right) \simeq T C\left(\mathbb{Z}_{p}^{\wedge}, p\right)$ (see for example $[13$, thm. 6.1]), and it follows that the map $\pi_{i} T C\left(\mathbb{Z}_{p}^{\wedge}, p\right) \rightarrow T C\left(\mathbb{Z} / p^{n}, p\right)$ is onto. In [4] Bökstedt and Madsen have computed $\pi_{*} T C\left(\mathbb{Z}_{p}^{\wedge}, p\right)$. In the low degrees we are interested in it is $\mathbb{Z}_{p}^{\wedge}$ in odd degrees and 0 in even strictly positive degrees. It follows that the group $\pi_{i} T C\left(\mathbb{Z} / p^{n}, p\right)$ is cyclic. Using the cofibration displayed in the proof of the above lemma and the computation of $\pi_{*} T C(\mathbb{Z} / p, p)$ given in [13] we can by induction prove that

$$
\pi_{i} T C\left(\mathbb{Z} / p^{n}, p\right) \cong \begin{cases}0 & \text { if } 0<i \leq p-3 \text { even } \\ \mathbb{Z} / p^{j(n-1)} & \text { if } i=2 j-1 \leq p-3 .\end{cases}
$$

The statement of corollary 7.4 now follows from McCarthy's theorem 6.2 and from Quillen's computation of $K(\mathbb{Z} / p)$ in [23].

\section{References}

[1] J. E. Aisbett, E. Lluis-Puebla and V. Snaith, On $K_{*}(Z / n)$ and $K_{*}\left(F_{q}[t] /\left(t^{2}\right)\right)$. Mem. Amer. Math. Soc. 57 (1985), no. 329.

[2] M. Bökstedt, Topological Hochschild homology, preprint Bielefeld 1986.

[3] M. Bökstedt, W.C. Hsiang and I. Madsen, The cyclotomic trace and algebraic $K$-theory of spaces, Invent. Math. 111 (1993), no. 3, 465-540.

[4] M. Bökstedt and I. Madsen, Topological cyclic homology of the integers, Asterisque 226 (1994), 57-143.

[5] A.K. Bousfield and E.M. Friedlander, Homotopy theory of $\Gamma$-spaces, spectra and bisimplicial sets, Lecture Notes in Math., Vol. 658, Springer (1978), 80-130.

[6] A.K. Bousfield and D.M. Kan, Homotopy limits, completions and localizations, Lecture Notes in Math., Vol. 304, Springer, Berlin (1972).

[7] M. Brun, Topological Hochschild homology of $\mathbb{Z} / p^{n}$, J. Pure Appl. Algebra 148 (2000), no. 1, 29-76.

[8] H. Cartan and S. Eilenberg, Homological algebra, reprint of the 1956 original, Princeton Landmarks in Mathematics, Princeton University Press, Princeton, NJ (1999).

[9] R. Cohen and J. D. S. Jones Algebraic K-theory of spaces and the Novikov conjecture, Topology 29 (1990), no. 3, 317-344. 
[10] L. Evens and E. Friedlander, On $K_{*}\left(\mathbb{Z} / p^{2}\right)$ and related homology groups, Trans. AMS 270 (1982) 1-46.

[11] T.G. Goodwillie, Relative algebraic $K$-theory and cyclic homology, Ann. Math. (2) 124 (1986), no. 2, 347-402.

[12] T.G. Goodwillie, Lectures notes on TC and the cyclotomic trace, MSRI preprint.

[13] L. Hesselholt and I. Madsen, On the $K$-theory of finite algebras over Witt vectors of perfect fields, Topology 36 (1997), no. 1, 29-101.

[14] L.G. Lewis, J.P. May and M. Steinberger, Stable equivariant homotopy theory, Lecture Notes in Math., Vol. 1213, Springer, Berlin.

[15] J.L. Loday, Cyclic Homology, Grundlehren der mathematischen Wissenschaften 301, Springer, Berlin (1992).

[16] M. Lydakis, Smash products and Gamma-spaces, Math. Proc. Camb. Phil. Soc. 126 (1999), no. 2, 311-328.

[17] I. Madsen, Algebraic K-theory and traces, Current developments in mathematics, 1995 (Cambridge, MA), 191-321, Internat. Press, Cambridge, MA, 1994.

[18] W. S. Massey, Exact couples in algebraic topology. I, II. Ann. of Math. (2) 56, (1952). 363-396.

[19] R. McCarthy, Relative algebraic $K$-theory and topological cyclic homology, Acta Math. 179 (1997), no. 2, 197-222.

[20] T. Pirashvili and F. Waldhausen, Mac Lane homology and topological Hochschild homology, J. Pure Appl. Algebra 82 (1992), no. 1, 81-98.

[21] S. Priddy, On a conjecture concerning $K_{*}\left(Z / p^{2}\right)$, Algebraic $K$-theory, Evanston 1980 (Proc. Conf., Northwestern Univ., Evanston, Ill., 1980), pp. 338-342, LNM 854, Springer, Berlin, 1981.

[22] D.G. Quillen, Homotopical Algebra, Lecture Notes in Math., Vol. 43, Springer (1967).

[23] D.G. Quillen, On the cohomology and $K$-theory of the general linear group over a finite field, Ann. Math. (2) 96 (1972), 552-586.

[24] G. Segal, Categories and cohomology theories, Topology 13 (1974), 293-312.

[25] G.W. Whitehead, Elements of Homotopy Theory, Graduate Texts in Mathematics 61, Springer, New York (1978).

Institut de Recherche Mathematique Avancée

CNRS et Université Louis Pasteur, 7 rue R. Descartes

67084 Strasbourg Cedex, France

Email: brun@math.u-strasbg.fr

Received: 17 October $2000 \quad$ Revised: 16 March 2001 